\title{
The field surrounding NGC 7603: Cosmological or non-cosmological redshifts?
}

\author{
M. López-Corredoira ${ }^{1}$ and C. M. Gutiérrez ${ }^{2}$ \\ 1 Astronomisches Institut der Universität Basel. Venusstrasse 7. 4102 Binningen, Switzerland \\ 2 Instituto de Astrofísica de Canarias, 38205 La Laguna, Tenerife, Spain
}

Received 2 September 2003 / Accepted 31 December 2003

\begin{abstract}
We present new observations of the field surrounding the Seyfert galaxy NGC 7603, where four galaxies with different redshifts - NGC $7603(z=0.029)$, NGC 7603B $(z=0.057)$ and two fainter emission line galaxies $(z=0.245$ and $z=0.394)$ - are apparently connected by a narrow filament, leading to a possible case of anomalous redshift. The observations comprise broad and narrow band imaging and intermediate resolution spectroscopy of some of the objects in the field. The new data confirm the redshift of the two emission-line objects found within the filament connecting NGC 7603 and NGC 7603B, and settles their type with better accuracy. Although both objects are point-like in ground based images, using HST archive images we show that the objects have structure with a $F W H M=0.3-0.4$ arcsec. The photometry in the $R$-band obtained during three different campaigns spread over two years does not show any signs of variability in these objects above $0.3-0.4 \mathrm{mag}$. All the above information and the relative strength and width of the main spectral lines allow us to classify these as HII galaxies with very vigorous star formation, while the rest of the filament and NGC 7603B lack star formation. We delineate the halo of NGC 7603 out to $26.2 \mathrm{mag} / \mathrm{arcsec}^{2}$ in the Sloan $r$ band filter and find evidence for strong internal distortions. New narrow emission line galaxies at $z=0.246,0.117$ and 0.401 are also found at respectively $0.8,1.5$ and 1.7 arcmin to the West of the filament within the fainter contour of this halo. We have studied the spatial distribution of objects in the field within 1.5 arcmin of NGC 7603. We conclude that the density of QSOs is roughly within the expected value of the limiting magnitude of our observations. However, the configuration of the four galaxies apparently connected by the filament appears highly unusual. The probability of three background galaxies of any type with apparent $B$-magnitudes up to 16.6, 21.1 and 22.1 (the observed magnitudes, extinction correction included) being randomly projected on the filament of the fourth galaxy (NGC 7603) is $\approx 3 \times 10^{-9}$. Furthermore, the possible detection of very vigorous star formation observed in the HII galaxies of the filament would have a low probability if they were background normal-giant galaxies; instead, the intensity of the lines is typical of dwarf HII galaxies. Hence, a set of coincidences with a very low probability would be necessary to explain this as a fortuitous projection of background sources. Several explanations in terms of cosmological or non-cosmological redshifts are discussed.
\end{abstract}

Key words. galaxies: individual: NGC 7603 - galaxies: statistics - galaxies: peculiar - galaxies: starburst cosmology: distance scale

\section{Introduction}

\subsection{Anomalous redshift problem}

The problem of the apparent optical associations of galaxies with very different redshifts, the so-called anomalous redshifts (Narlikar 1989; Arp 1987, 1998), is old but still unresolved. Although surprisingly ignored by most of the astronomical community, there is increasing evidence of examples of such anomalies. Statistical evidence has grown for such associations over the last 30 years (Burbidge 1996, 2001). For instance, all non-elliptical galaxies brighter than 12.8 mag with apparent companion galaxies have been examined (Arp 1981), and 13 of the 34 candidate companion galaxies were found to have QSOs with higher redshift. Given a probability of less than 0.01 per

Send offprint requests to: M. López-Corredoira, e-mail: cgc@ll.iac.es galaxy, the global probability of this to be chance is $\sim 10^{-17}$. Bias effects alone cannot be responsible for these correlations (Burbidge 2001; Hoyle \& Burbidge 1996; Benítez et al. 2001). Weak gravitational lensing by dark matter has been proposed as the cause of these correlations (Gott \& Gunn 1974; Schneider 1989; Wu 1996; Burbidge et al. 1997), although this seems to be insufficient to explain them (Burbidge et al. 1997; Burbidge 2001; Benítez et al. 2001; Gaztañaga 2003; Jain et al. 2003), and cannot work at all for the correlations with the brightest and nearest galaxies. The statistical relevance of these associations is still a matter of debate (Sluse et al. 2003).

A recent compilation of associations of galaxies-QSOs has been presented by Burbidge (1996). Some remarkable cases of apparent associations between objects with different redshift are Arp 220 (Ohyama et al. 1999; Arp et al. 2001), NGC 1068 (Burbidge 1999a; Bell 2002a), NGC 3067 (Carilli et al. 1989; Carilli \& van Gorkom 1992), NGC 3628 (Arp et al. 2002), 
NGC 4258 (Pietsch et al. 1994; Kondratko et al. 2001), NGC 4319 (Sulentic \& Arp 1987), etc. Some of these may be just fortuitous cases in which background objects are close to the foreground galaxy, although the statistical mean correlations remain to be explained, and some cases alone have very small probability of being a projection of background objects.

Associations of galaxies with different redshifts might also take place: forty-three systems among the hundred Hickson (1982) groups of galaxies (compact groups of galaxies containing four to six members) have one redshift very different from the mean of the others (Sulentic 1997). For instance, Stephan's quintet (Moles et al. 1998; Gutiérrez et al. 2002), the chain VV172 (Arp 1987; Narlikar 1989), etc. Although the numbers, sizes, magnitudes and morphological types of the discordant redshift members might agree with a scenario of chance projections, the distribution of positions in quintets is more centrally concentrated than expected in such a scenario (Mendes de Oliveira 1995). This author claims that compact groups might act as gravitational lenses and therefore explain the difference in concentration, but this remains to be justified.

To explain these associations Hoyle et al. (1993) proposed new physics in which part of the measured redshifs are not caused by the expansion of the Universe. Other theories have been proposed too (see Sect. 5.3). We are carrying out a series of observations of some of the suspicious systems to clarify the problem (Gutiérrez et al. 2002; López-Corredoira \& Gutiérrez 2002; Gutiérrez \& López-Corredoira 2004; and this paper). In particular, this paper is about the system of NGC 7603 and the surrounding objects.

\subsection{NGC 7603}

The main galaxy, NGC 7603, is a broad line Seyfert I galaxy with $z=0.0295$ and $B=14.04 \mathrm{mag}$ (de Vaucouleurs et al. 1991). This galaxy has been studied mainly in relation to its variability, which was discovered by Kopylov et al. (1974), and Tohline \& Osterbrock (1976). Kollatschny et al. (2000) have presented the results of an extensive programme to study the line and continuum variability over a period of twenty years. They detected spectral variations on timescales from months to years. The variability observed is 5-10 in the intensity in the Balmer and Helium lines and in the continuum. From the perspective of the Eigenvector 1 parameter space for AGNs (Sulentic et al. 2000, 2002), the Balmer lines are unusually broad and show a very complex structure. The Balmer lines are blueshifted relative to the local "rest frame" of the AGN by between 1000 and $2000 \mathrm{~km} \mathrm{~s}^{-1}$. Less than $5 \%$ of AGN show such characteristics. Such lines are more common in radio-loud quasars, where one sees ejected synchrotron lobes. It shows unusually strong FeII emission for an AGN with such broad lines (Goodrich 1989; Kollatschny et al. 2000).

The system around NGC 7603 is very interesting because it is among the cases (Arp 1980) with some filamentary structure joining galaxies with different redshift. Arp (1971, 1975, 1980) has claimed that the compact member has somehow been ejected from the bigger object. NGC 7603 and its filament are so distorted that significant tidal disturbance can be reasonably assumed, without a clear candidate for the companion galaxy producing the tides (see Sect. 3.1). Another fact that has attracted attention (Arp 1971, 1975; Sharp 1986) is the proximity of NGC 7603B, a spiral galaxy with higher redshift $(z=0.0569)$ located 59 arcsec to the SE of NGC 7603. The angular proximity of both galaxies and the apparently luminous connection between them, makes the system an important example of an anomalous redshift association. Hoyle (1972) has pointed out that NGC 7603 is one of the most strange cases, and which needs a non-standard theory to be explained. Apart from the above facts there are also two in principle point-like objects superimposed on the filament that apparently connects both galaxies.

In López-Corredoira \& Gutiérrez (2002, hereafter Paper I) we presented intermediate resolution spectra of the filament and the two objects mentioned (see Fig. 1 of Paper I). From several absorption lines we estimated the redshift of the filament apparently connecting NGC 7603 and NGC 7603B as $z=0.030$, very similar to the redshift of NGC 7603 and probably associated with this galaxy. We identified several emission lines in the spectra of the two knots and from the emission lines of $\mathrm{H} \beta$, OII (3727 $\AA$ ) and OIII (4959 and $5007 \AA$ ) we determined their redshifts, obtaining 0.39 and 0.24 for the objects closer and farther from NGC 7603 respectively. The two objects might be QSOs or HII-galaxies. The spectra of Paper I had not enough resolution to determine their nature definitively (since we used a wide slit) and the seeing conditions limited the possibility of seeing structure under 1 arcsec in these objects. We planned new observations with the aim of answering the following questions: i) What is the nature of the two knots in the filament? ii) Are there any other high redshift objects in the halo surrounding NGC 7603? And iii) are there any clues in the surrounding field that help us understand the nature of this apparent association?

This paper contains the analysis of these new observations and is structured as follows: Sect. 2 presents the details of the observations and data reduction. Section 3 presents the observed images, and the main features discovered in each component. Section 4 presents the spectroscopy of some sources. Section 5 calculates the probabilities of the observed configuration being an accidental projection of background galaxies, and discusses the results presenting some possible physical scenarios to explain them. A summary of the main results is given in Sect. 6.

\section{Observations}

The observations presented here comprise narrow and broad band imaging, and spectroscopy with intermediate resolution. These observations were taken at the $\mathrm{IAC}^{1} 0^{1}, \mathrm{NOT}^{2}, \mathrm{WHT}^{3}$

1 The telescope is at the Spanish Teide Observatory on the island of Tenerife and is operated by the Instituto de Astrofísica de Canarias.

2 The Nordic Optical Telescope (NOT) is operated on the island of La Palma jointly by Denmark, Finland, Iceland, Norway, and Sweden, in the Spanish Observatorio del Roque de los Muchachos of the Instituto de Astrofísica de Canarias.

3 The William Herschel Telescope (WHT) is operated on the island of La Palma by the Isaac Newton Group of Telescopes. 
Table 1. Observations.

\begin{tabular}{|c|c|c|c|c|}
\hline Telescope and instrument & Epoch & Mode & Exposure time & Seeing \\
\hline \multirow[t]{2}{*}{ IAC80 (0.8 m) CCD } & Jul. 8-00/Aug. 17-01 & Image, narrow-fi.IAC39-6767 A & $30000 \mathrm{~s}$ (dark) & $1.8^{\prime \prime}$ \\
\hline & Aug. 6-00/Jul. 22, Aug. 15-01 & Image, narrow-fi.IAC35-6931 & 52834 s (grey) & $1.8^{\prime \prime}$ \\
\hline \multirow[t]{10}{*}{ NOT (2.6 m) ALFOSC } & 2000 June 13 & Image, narrow-fi.IAC39-6767 & $900 \mathrm{~s}$ (dark) & $1^{\prime \prime}$ \\
\hline & 2000 June 13 & Image, narrow-fi.IAC35-6931 & $900 \mathrm{~s}$ (bright) & $1^{\prime \prime}$ \\
\hline & 2000 June 13 & Image, $R$-Bessel band & $900 \mathrm{~s}$ (dark) & $1^{\prime \prime}$ \\
\hline & 2001 August 12 & Image, $R$-Bessel band & $300 \mathrm{~s}$ (dark) & $1.5^{\prime \prime}-2.0^{\prime \prime}$ \\
\hline & 2002 November 30 & Image, $R$-Bessel band & $900 \mathrm{~s}$ (dark) & $1.5^{\prime \prime}-2.0^{\prime \prime}$ \\
\hline & 2002 November 30 & Image, $u$-Sloan band & 3200 s (dark) & $1.5^{\prime \prime}-2.0^{\prime \prime}$ \\
\hline & 2002 November 30 & Image, $g$-Sloan band & $1200 \mathrm{~s}$ (dark) & $1.5^{\prime \prime}-2.0^{\prime \prime}$ \\
\hline & 2002 November 30 & Image, $i$-Sloan band & $900 \mathrm{~s}$ (dark) & $1.5^{\prime \prime}-2.0^{\prime \prime}$ \\
\hline & 2002 December 2 & Image, $r$-Sloan band & $1200 \mathrm{~s}$ (non-phot.) & $1.5^{\prime \prime}-2.0^{\prime \prime}$ \\
\hline & 2002 December 3 & Image, $r$-Sloan band & $4200 \mathrm{~s}$ (dark) & $1.5^{\prime \prime}-2.0^{\prime \prime}$ \\
\hline (only Paper I) $-->$ & August 12, 2001 & Spectr.1.slit, gr.\#4, ap.5" & 14225 s (dark) & $1.5^{\prime \prime}-2.0^{\prime \prime}$ \\
\hline \multirow[t]{3}{*}{ WHT (4.2 m) ISIS red arm } & 2002 December 28 & Spectr.1.slit, gr.R158R, ap.1.2" & $5400 \mathrm{~s}(\operatorname{Pos} 1)$ & - \\
\hline & 2002 December 29 & Spectr.1.slit, gr.R158R, ap.1.5" & $5400 \mathrm{~s}(\operatorname{Pos} 2)$ & - \\
\hline & 2002 December 292002 & Spectr.1.slit, gr.R158R, ap.1.5" & $1800 \mathrm{~s}(\operatorname{Pos} 3)$ & - \\
\hline HST WFPC2 & 1994 July 3 & Image, filter $F 606 \mathrm{~W}$ & $500 \mathrm{~s}$ & - \\
\hline
\end{tabular}

telescopes, and from the HST archive ${ }^{4}$. Table 1 presents a summary of the observations.

We wanted to check for the presence of $\mathrm{H} \alpha$ emission in the filament connecting NGC 7603 and NGC 7603B as well as in the galaxies themselves. NII (6583 $\AA$ ) is also interesting and might be stronger if e.g. shocks were involved; it would be observed in narrow filters of FWHM $50 \AA$ centered at $\mathrm{H} \alpha$ emission. During several campaigns in 2000 and 2001 we obtained imaging at the IAC 80 and NOT with the IAC39 and IAC35 filters which are centred on 6767 and $6931 \AA$ and which match the $\mathrm{H} \alpha$ line at velocities of 9372 and $16870 \mathrm{~km} \mathrm{~s}^{-1}$ respectively and have a $F W H M$ equivalent to $2000 \mathrm{~km} \mathrm{~s}^{-1}$. These ranges in velocity correspond to the redshifts of NGC 7603 and NGC 7603B respectively. The images were reduced using a standard procedure that comprises bias subtraction, flatfield correction, shifting and co-addition of individual exposures. The continuum in each case was subtracted using a resampled and scaled image (in order to have the same resolution of the IAC80: $0.435 \mathrm{arcsec}_{\mathrm{pixel}}{ }^{-1}$ ) in the $R$ band taken on 2000 June 13th at the NOT.

With the broad band images we wanted to delineate in detail the halo of the system NGC 7603-NGC 7603B, to detect other possible candidates in the field and measure their colours, and to constrain possible variability of the two objects in the filament. For the study of the variability we took several images in the $R$ band (Bessel) in the period 2000-2002. For the remaining tasks, apart from the $R$ filter, we observed the field with the Sloan $u, g, r$, and $i$ filters. In all cases we used the NOT with the ALFOSC instrument. The images were reduced following the standard procedure mentioned above. The conditions were photometric in all runs except on December 2. For the 2000 June 13th observation, several Landolt calibration fields (Landolt 1992) were observed. The observations in the

${ }^{4}$ Based on observations made with the NASA/ESA Hubble Space Telescope, obtained from the data archive at the Space Telescope Science Institute. STScI is operated by the Association of Universities for Research in Astronomy, Inc. under NASA contract NAS 5-26555. other two runs with the $R$ filter at the NOT (2001 August 12th and 2002 November 30) were relative calibrated with respect to this using using eight stars in the field. For the Sloan filters, we have calibrated with some stars from the list given by Smith et al. (2002).

Because of limiting atmospheric conditions, we could not see details below 1 arcsec resolution from the ground telescopes images. Therefore, Hubble Space Telescope archive were also used to obtain a high spatial resolution of the objects embedded in the filament. These data come from the HST Proposal 5479 made by Matthew Malkan, which was used to produce the paper Malkan et al. (1998). The image, although less deep (exposure time: $500 \mathrm{~s}$ ), allows us to see small scale details of some interesting objects, since this includes the filament connecting NGC 7603 and NGC 7603B.

We obtained spectroscopy in two campaigns, the first at the NOT using ALFOSC (presented in Paper I and not considered here) and the second nearly a year and a half later using ISIS at the WHT in order to get further and better spectra than in Paper I and to study other objects in the field. At the WHT we put the slit in three different positions to optimize the observation of the objects within the filament and several other objects that were selected according to their colours (see Sect. 3.2). The grism used was R158R. We took Tungsten, and $\mathrm{Cu}-\mathrm{Ne}$ and $\mathrm{Cu}-\mathrm{Ar}$ calibration lamps for flat-field correction and spectral calibration respectively. The data were bias subtracted. After some tests we decided not apply any flat field correction because such corrections would require prohibitive exposure times with the Tungsten lamp on the blue side of the spectrum and this correction is very small $(\sim 1 \%)$ in the red part of the spectrum. The FWHM measured of the lines is $8 \AA$ for the first position, and $20 \AA$ for the second and third position. We extracted the spectra using the task apall of IRAF ${ }^{5}$. The data are sampled at $1.62 \AA /$ pixel and cover from $2810 \AA$ to $10450 \AA$.

\footnotetext{
5 IRAF is Image Reduction and Analysis Facility, written and supported by the IRAF programming group at the National Optical Astronomy Observatories (NOAO) in Tucson, Arizona.
} 


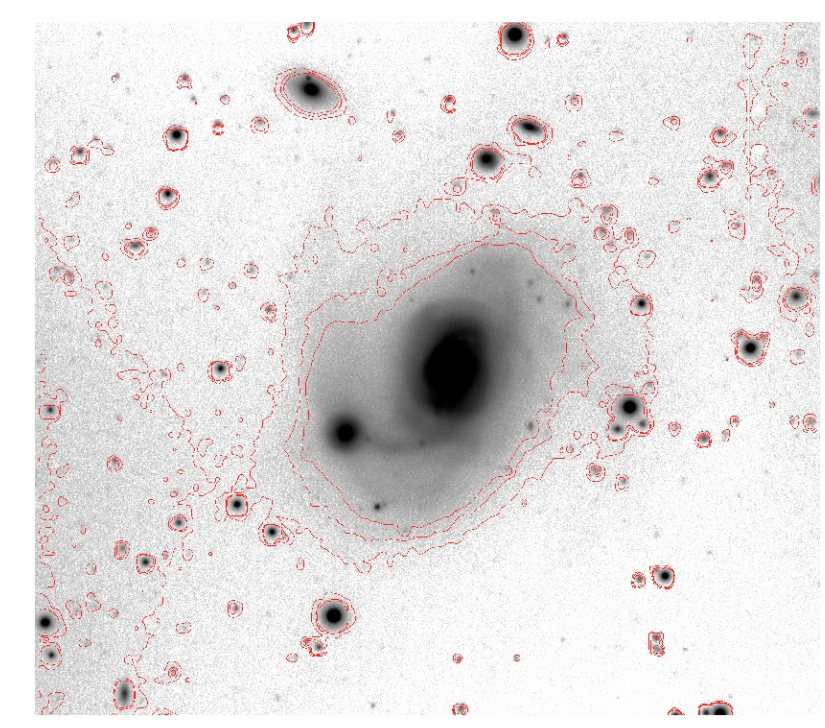

Fig. 1. A grey scale and contour image in the $R$ band of the region around the galaxy NGC 7603. The contours correspond to isophotes 24.8, 25.3 and $26.2 \mathrm{mag} / \mathrm{arcsec}^{2}$.

However, due to the response of the grism, the sensitivity of the first 1000 pixels (below $4400 \AA$ ) or the last 700 pixels (over $9300 \AA$ ) is very poor and have not been used in any of the analyses.

\section{Imaging}

\subsection{Morphology and surface photometry}

Figure 1 shows the $R$ band image obtained combining the different observations in this band (see also Fig. 1 of Kollatschny et al. 2000). The figure presents the grey-scale and isophotal maps in this filter. The high emission due to the activity of the galaxy NGC 7603 saturates the image in the central part of this galaxy. The system NGC 7603-NGC 7603B appears to be surrounded by a diffuse halo that we have been able to delineate out to $26.2 \mathrm{mag} / \operatorname{arcsec}^{2}$ in the Sloan $r$-band filter. Although this halo seems to be associated mostly with NGC 7603, it is not symmetric with respect to this galaxy. There is evidence of a fainter extension tail in northern direction. The last isophote of the halo is also asymmetric to the West, possibly including a counter arm of the bright filament between NGC 7603 and NGC 7603B. The halo+filament between NGC 7603 and NGC 7603B shows up clearly and has a maximum brightness of $22.9 \mathrm{mag} / \mathrm{arcsec}^{2}$ in the Sloan $r$-band filter, while the halo has a brightness near the filament of $23.4 \mathrm{mag} / \mathrm{arcsec}^{2}$. Therefore, the filament alone has around $24.0 \mathrm{mag} / \mathrm{arcsec}^{2}$. Another diffuse structure is seen apparently connecting NGC 7603 and NGC 7603B also, and situated to the South of the main filament. A point like object (\#17 of Fig. 4) situated in the southest point of this tail has been also observed spectroscopically (see below) resulting a local star.

Figure 2 shows a map of contours of the $\mathrm{H} \alpha$ emission in the IAC39 filter (centred on the redshift of the galaxy NGC 7603) once the continuum ( $R$-filter) is roughly subtracted. No emission was found in the IAC35 filter (centred on the redshift of the galaxy NGC 7603B), either in NGC 7603B or in the

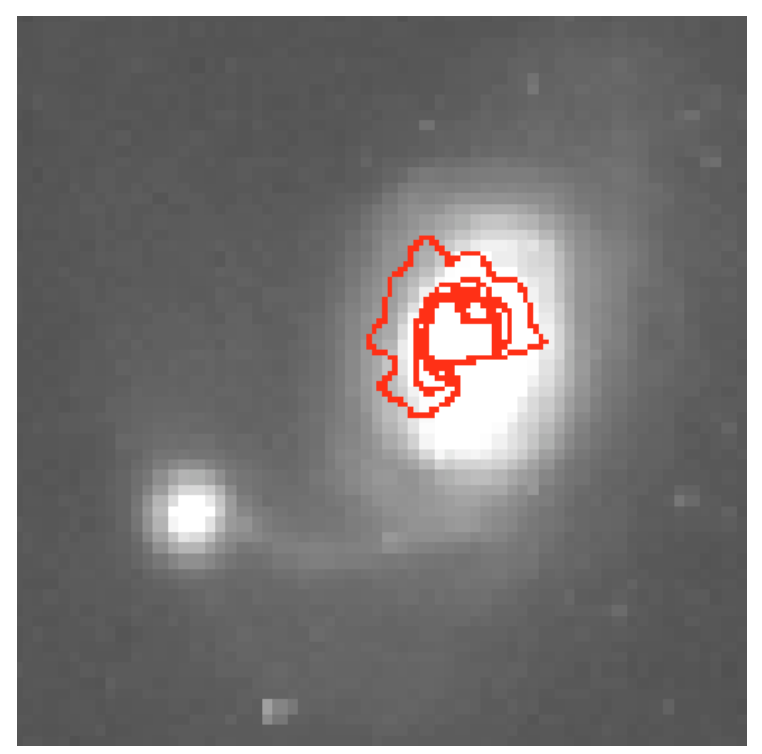

Fig. 2. A grey-scale $R$ band image and contours corresponding to $\mathrm{H} \alpha$ emission at the redshift of the galaxy NGC 7603.

filament. Only the nucleus of the NGC 7603 (IAC39 filter) shows some emission, as expected from a Seyfert 1 galaxy. No stripped emission regions (as found, for instance, in the stripping event in Stephan's quintet; Sulentic et al. 2001; Gutiérrez et al. 2002) were observed. This absence of $\mathrm{H} \alpha$ emission lines in NGC 7603B has already been pointed out by Sharp (1986). The non-detection of emission lines is not proof against the existence of a physical connection. In interactions and ejections with a larger galaxy, the gas is often stripped out of a stellar system (Rose et al. 2001); so the lack of emission lines could be taken as an indication of interaction rather than non interaction (pointed out by Sharp 1986).

Figures 1-3 show that NGC 7603 and its filament are apparently distorted by significant tidal interaction. The existence of the filament is also a possible sign of tidal interaction or a debris from satellite disruption (Johnston et al. 2001). The fainter southern filament (the one which crosses object \#17) and the red fringe embedded in NGC 7603 (red colour in Fig. 3; due possibly to dust) reinforces the scenario of tidal debris. The colours of the filament connecting NGC 7603 and NGC 7603B are $(g-r) \approx 0.95$ (equivalent to $(B-V) \approx 1.15$, similar to the bridge of the interacting system Arp 96; Schombert et al. 1990) and $(u-g) \approx 1.5$ (like the outer region of NGC 7603). Finally, we do not detect emission lines in that filament.

\subsection{The neighborhood of NGC 7603}

First, we looked for QSOs, since they are typical objects among anomalous redshift candidates. We try to identify QSOs with $z<2.5$ in the field using the multicolour criteria proposed in the analysis of the $2 \mathrm{dF}$ Survey (Boyle et al. 2000; Meyer et al. 2001). This criterion, converted into Sloan filters through the relations between the UBVRI Johnson filters and ugri Sloan filters given by Smith et al. (2002), and the relation between the photographic filter $b_{j}$ and Johnson 
filters: $b_{j}=B-0.28(B-V)$ (Meyer et al. 2001); (we adopt the approximation of $U, R$ photographic-filters equivalent to $U, R$ Johnson-filters) is:

$(g-r)<\left\{\begin{array}{ll}\frac{0.552-(u-g)}{0.381}, & (u-g)<0.452 \\ \frac{0.921-(u-g)}{1.793}, & 0.452<(u-g)<1.197 \\ -0.154, & (u-g)>1.197\end{array}\right\}$.

The completeness is quite high; this criterion covers 80-90\% of all QSOs (Meyer et al. 2001). The principal contamination comes from Galactic stars, subdwarfs and white dwarfs, and blue compact emission-line galaxies (Croom et al. 2001). The total fraction of the contaminant sources is $\approx 45 \%$ (Croom et al. 2001). Therefore, the total number of QSOs will be $\approx 2 / 3$ times the number of objects that follow the criterion of Eq. (1). This number is slightly different when the range of magnitudes is different from those of the $2 \mathrm{dF}$ survey, but not by too much.

We proceed as follows: first we select all objects in the field detected in the $u$ filter. There are 38 objects in this filter, including the two knots in the filament, but excluding NGC 7603 and NGC 7603B. We used the software "Sextractor" (Bertin $\&$ Arnouts 1996) to measure the photometry of these objects in the $u, g, r, i$ filters. For the two objects in the filament first we tried to subtract the contribution of the filament by a twodimensional surface fit. Although we tried with different functions, ranges, etc, the result was not satisfactory partly owing to the presence of the two main galaxies, but the accuracy in the estimation of the magnitudes for these two objects is good enough within an uncertainty of $\sim 0.2 \mathrm{mag}$. Also, the photometry of object \#35 was done separately because it was embedded in the halo of NGC 7603, taking special care in sky subtraction. It is noteworthy that object \#35 has a quite high value of $(g-r)=1.8$, while $(u-g)=0$; although it does not follow Eq. (1), it may be a unusual object because of its colours. Table 2 presents the results on the photometry in $u, g, r$ and $i$ of all the objects, whose positions are shown in Fig. 4 . We have no $u$ magnitude for object \#1 because it is too faint in this filter. Figure 5 presents a colour-colour diagram for these objects, and the regions in which QSOs are expected. We see that objects \#19, \#23 and \#36 follow the criterion of Eq. (1), which is indicated in Fig. 5.

\subsubsection{Galaxies in/behind the filament}

We now examine objects 1,2 , either embedded in the filament that joins NGC 7603 and NGC 7603B or behind the filament. The two objects appear point like in our deep image in the $R$ band (see above). The field of the filament was observed in the $F 606 W$ filter with the Hubble Space Telescope. It does not cover the other three narrow emission line galaxies, but we can examine how extended the objects are in the filament. Figure 6 shows this image. The field is centred on the filament between NGC 7603 and NGC 7603B and clearly shows the two objects within it. Both of them appear as extended objects; this is specially clear for object \#1 (the one closer to NGC 7603). The figure also shows a contour plot of both objects which confirms the visual impression of both as extended objects. The $F W H M$ s of objects 1 and 2 are $\sim 0.3$ and $\sim 0.4$ arcsec respectively, which
Table 2. Magnitudes of the objects in the field of NGC 7603 derived using "Sextractor" (except those marked with *, which were derived separately with "phot" taking care of the filament/halo subtraction and are affected by an error of at least $0.2 \mathrm{mag}$ ). Last column points out whether they are extended "E" (as far as we can see from the available images and spectra; some further faint objects which look point-like might be extended too).

\begin{tabular}{rllllr}
\hline \hline$\#$ & $u$ & $g$ & $r$ & $i$ & Ext. \\
\hline 1 & - & $23.1^{*}$ & $21.7^{*}$ & $21.6^{*}$ & $\mathrm{E}$ \\
2 & $23.8^{*}$ & $22.9^{*}$ & $22.2^{*}$ & $21.8^{*}$ & $\mathrm{E}$ \\
3 & 20.7 & 19.4 & 18.7 & 18.6 & $\mathrm{E}$ \\
4 & 20.9 & 18.1 & 16.6 & 16.0 & \\
5 & 21.8 & 19.7 & 18.9 & 18.8 & \\
6 & 18.6 & 17.3 & 16.7 & 16.7 & \\
7 & 22.6 & 21.0 & 20.4 & 20.0 & $\mathrm{E}$ \\
8 & 17.6 & 16.0 & 15.3 & 15.1 & \\
9 & 22.9 & 21.1 & 20.5 & 20.2 & $\mathrm{E}$ \\
10 & 21.5 & 21.2 & 20.4 & 20.5 & \\
11 & 23.7 & 21.8 & 20.5 & 20.2 & \\
12 & 23.3 & 22.4 & 21.3 & 21.2 & \\
13 & 19.7 & 18.6 & 18.1 & 18.2 & \\
14 & 23.1 & 21.2 & 20.5 & 20.4 & $\mathrm{E}$ \\
15 & 21.2 & 20.1 & 19.6 & 19.6 & \\
16 & 21.4 & 20.5 & 20.0 & 19.8 & \\
17 & 20.6 & 20.0 & 19.4 & 19.5 & \\
18 & 21.1 & 19.4 & 18.5 & 18.6 & \\
19 & 22.5 & 22.4 & 22.0 & 21.6 & \\
20 & 17.6 & 16.4 & 15.7 & 15.8 & \\
21 & 21.9 & 20.5 & 19.5 & 19.2 & $\mathrm{E}$ \\
22 & 22.0 & 21.4 & 19.9 & 19.7 & $\mathrm{E}$ \\
23 & 21.6 & 21.6 & 20.8 & 20.6 & $\mathrm{E}$ \\
24 & 19.4 & 17.9 & 17.0 & 17.0 & \\
25 & 20.7 & 19.7 & 19.1 & 19.2 & \\
26 & 22.4 & 19.3 & 17.8 & 17.2 & \\
27 & 21.9 & 21.2 & 20.4 & 20.0 & $\mathrm{E}$ \\
28 & 20.3 & 18.6 & 17.7 & 17.5 & $\mathrm{E}$ \\
29 & 19.3 & 17.3 & 16.2 & 16.0 & $\mathrm{E}$ \\
30 & 19.0 & 17.8 & 17.2 & 17.1 & $\mathrm{E}$ \\
31 & 23.0 & 20.2 & 18.8 & 18.4 & \\
32 & 21.3 & 20.2 & 19.6 & 19.3 & $\mathrm{E}$ \\
33 & 23.4 & 22.0 & 20.8 & 20.1 & $\mathrm{E}$ \\
34 & 23.8 & 22.3 & 21.0 & 20.1 & $\mathrm{E}$ \\
35 & $22.8^{*}$ & $22.8^{*}$ & $21.0^{*}$ & $21.6^{*}$ & \\
36 & 23.4 & 23.3 & 22.5 & - & \\
37 & 22.7 & 21.6 & 21.1 & 20.5 & \\
38 & 20.4 & 19.4 & 18.9 & 18.9 & \\
\hline & & & & &
\end{tabular}

is rather small to be measured in a ground-based telescope with seeing of 1 arcsec, and seems to indicate that they are extended, rather point-like objects.

The two objects in the filament are apparently slightly deformed, although the significance is not too high (the two lowest isocontours in Fig. 6 are $\sim 2 \sigma$ and $\sim 3.5 \sigma$ respectively over the average flux in the region). The tail of object \#1 in the northern part is warped pointing towards NGC 7603; and object \#2 has also a faint tail in the northern part.

With the $R$ band imaging we have studied the possible variability of these objects. In addition to the weakness of these objects, the presence of the filament makes the estimation of the magnitudes more difficult. We have calibrated with standard 


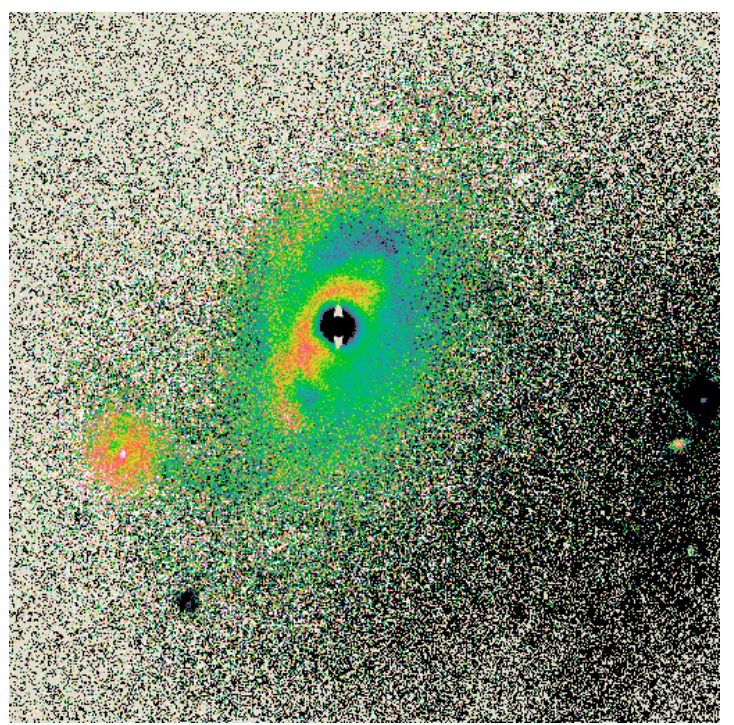

Fig. 3. Sloan $g-r$ colour of NGC7603. From bluer to redder colours (lower to higher values of $(g-r))$ : black-blue-green-red-white. The center of NGC 7603 is saturated. Noteworthy aspects are the red colour of an asymmetrical strip crossing NGC 7603, the young population (blue) at the north of NGC 7603 and the average (green) colour of the filament connecting NGC 7603 and NGC 7603B.

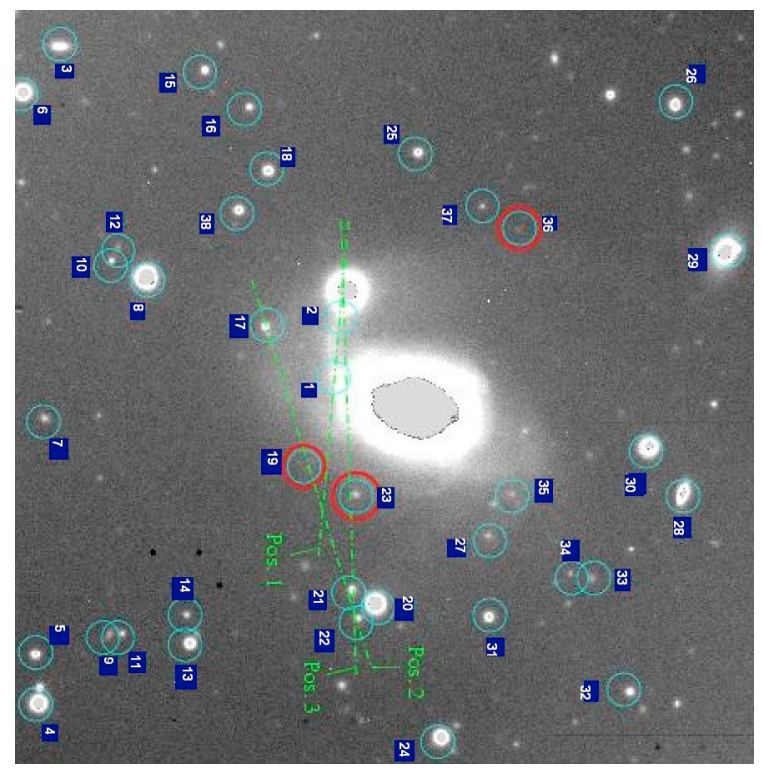

Fig. 4. Position of the sources in Table 2 (only sources with $m_{u} \leq 23.8$ except source \#1; NGC 7603 and NGC 7603B not included). With the double circle, we point out the three sources which follow Eq. (1), candidate QSOs by the multicolour selection. Dot-dashed lines represent the position of the three long slits placed in the field of NGC 7603 to obtain the spectra of some objects.

stars only the $R$ image taken on June 13, 2000, but we have performed differential photometry of the other images with eight bright stars in the field. According to the mentioned uncertainties, we conclude the absence of variability above $0.3-0.4$ mag.

Table 3 (Cols. 3 and 4) also shows the magnitudes in the various Sloan, Johnson and the $b_{j}$ photographic filters. Magnitudes in Johnson filters and $b_{j}$ photographic filter were
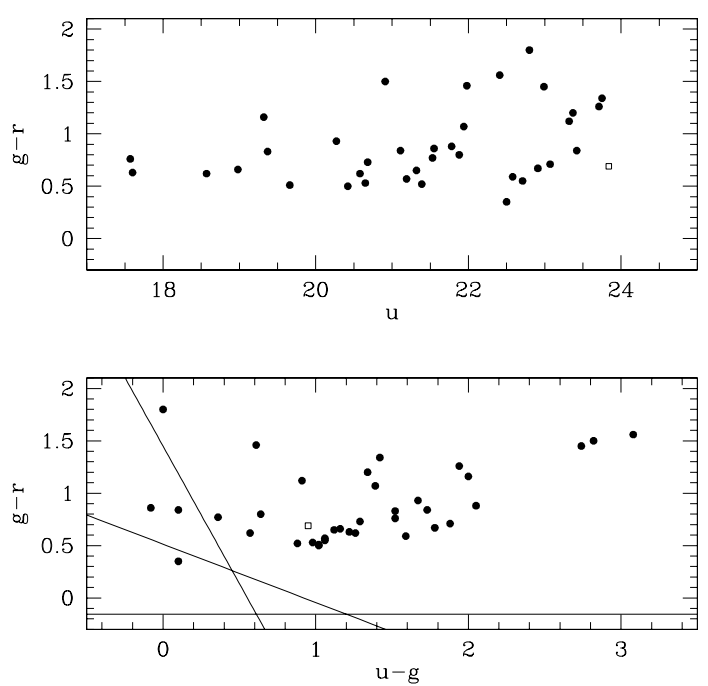

Fig. 5. Diagrams colour-apparent magnitude and colour-colour of objects that were selected in the field of NGC 7603 (Table 2). The open square represents object \#2. Object \#1 is not in the plots because we have not its $u$ magnitude. The lines indicate the limits of $(g-r)$ colour under which QSOs are likely to be found (there are 3 candidates).

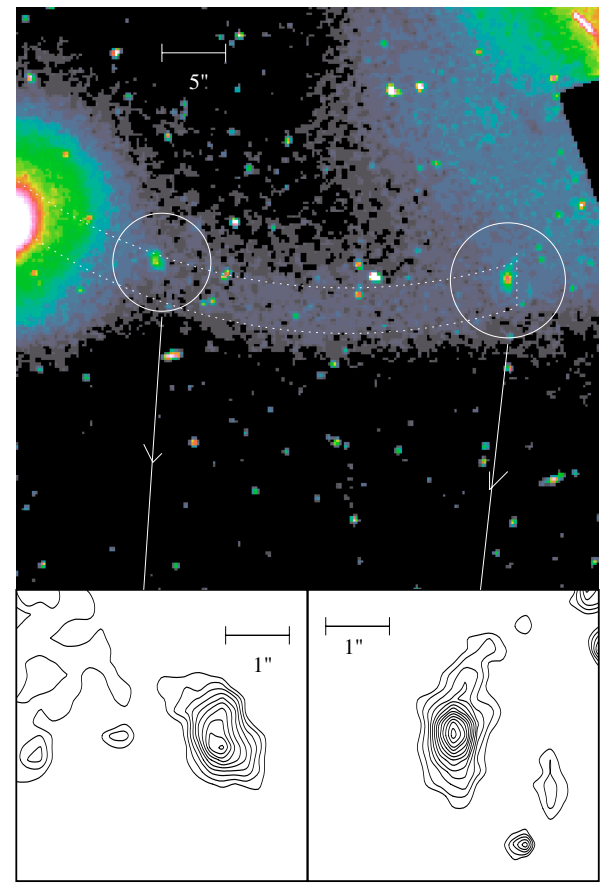

Fig. 6. HST image in the $F 606 \mathrm{~W}$ filter of the region centred on the filament between NGC 7603 and NGC 7603B. Also shown are the contours of the two objects in the filament. Note that there are many bad pixels/cosmic rays in the images that do not correspond to any object. The PSF is $\sim 0.1$ arcsec. Dotted lines show the area (around $140 \operatorname{arcsec}^{2}$ ) that we consider "filament" for the calculation of the probabilities in Sect. 5.2.2.

calculated from the magnitudes in Sloan filters for these objects through the relations between $U B V R I$ Johnson filters and ugri Sloan filters, like in Sect. 3.2. 
Table 3. Apparent magnitudes of objects in the filament between NGC 7603 and NGC 7603B. The $R$-Bessel magnitude was measured for three different epochs, the Sloan filters for only one epoch. Magnitudes in the Johnson filters and $b_{j}$ photographic filter were calculated from the magnitudes in the Sloan filters (see text). Columns 3 and 4 give the observed apparent magnitudes (uncertainty $\sim 0.2$ mag.). Columns 5 and 6 give the same magnitudes corrected of Galactic extinction (Schlegel et al. 1998) and filament extinction (by means of Eqs. (3) and (4)).

\begin{tabular}{ll|ll|ll}
\hline \hline Filter & Epoch of calculation & Mag \#1 & Mag \#2 & Mag corr. ext. \#1 & Mag corr. ext. \#2 \\
\hline Bessel $R$ & June 13th, 2000 & 21.20 & 21.70 & $19.5 \pm 0.7$ & $20.9 \pm 0.7$ \\
Bessel $R$ & August 12th, 2001 & 21.09 & 21.56 & $19.4 \pm 0.7$ & $20.8 \pm 0.7$ \\
Bessel $R$ & November 30th, 2002 & 21.29 & 21.87 & $19.6 \pm 0.7$ & $21.1 \pm 0.7$ \\
\hline Sloan $u$ & November 30th, 2002 & - & 23.84 & - & $22.2 \pm 1.4$ \\
Sloan $g$ & November 30th, 2002 & 23.08 & 22.89 & $20.4 \pm 1.1$ & $21.6 \pm 1.1$ \\
Sloan $r$ & December 3rd, 2002 & 21.51 & 22.03 & $19.7 \pm 0.7$ & $21.1 \pm 0.7$ \\
Sloan $i$ & November 30th, 2002 & 21.55 & 21.77 & $20.1 \pm 0.7$ & $21.1 \pm 0.7$ \\
\hline Johnson $U$ & calc. from Sloan filters & - & 23.35 & - & $21.9 \pm 1.4$ \\
Johnson $B$ & calc. from Sloan filters & 23.99 & 23.47 & $21.1 \pm 1.1$ & $22.1 \pm 1.1$ \\
Johnson $V$ & calc. from Sloan filters & 22.19 & 22.39 & $20.0 \pm 1.0$ & $21.3 \pm 1.0$ \\
Johnson $R$ & calc. from Sloan filters & 21.15 & 21.77 & $19.5 \pm 0.7$ & $21.0 \pm 0.7$ \\
Johnson $I$ & calc. from Sloan filters & 20.99 & 21.30 & $19.9 \pm 0.4$ & $20.8 \pm 0.4$ \\
\hline $\mathrm{b}_{j}$ photogr. & calc. from Johnson filters & 23.49 & 23.17 & $20.8 \pm 1.1$ & $21.9 \pm 1.1$ \\
\hline
\end{tabular}

Table 4. Spectral analysis. The error in the redshift is $\approx \pm 0.002$. The codes for the last two columns are: 0 -absorption emission line galaxy; 1-narrow emission line galaxy; 2-star; 3-contamination by the filament; 4-spectra very noisy.

\begin{tabular}{llllll}
\hline \hline Slit Pos. & Object & Spectral features $(>3 \sigma)$ & Redshift & Type & Notes \\
\hline 1,3 & NGC 7603B & CaH\&K, MgI, NaI, etc. & 0.056 & 0 & \\
1 & $\# 1$ & OII, H $\beta$, OIII, OI, H $\alpha$ & 0.394 & 1 & \\
1 & $\# 2$ & OII, NeIII, H $\beta$, OIII, H $\alpha$ & 0.245 & 1 & \\
1 & Filament & $\mathrm{H} \beta, \mathrm{MgI}, \mathrm{NaI}$ & 0.030 & Abs. & 4 , Paper I \\
2 & $\# 17$ & $\mathrm{H} \alpha, \mathrm{H} \beta$ & 0 & 2 & 3 \\
2 & $3.6^{\prime \prime}-\mathrm{WNW}$ of \#17 & - & - & - & 4 \\
2 & $\# 19$ & - & - & - & 4 \\
2,3 & $\# 21$ & OIII & & 1 & \\
2,3 & $\# 22$ & $\mathrm{OII}, \mathrm{H} \beta, \mathrm{H} \alpha$ & 0.117 & 1 & \\
3 & $\# 23$ & $\mathrm{H} \alpha$ & 0.401 & 1 & \\
\hline
\end{tabular}

\section{Spectroscopy}

The QSO candidates are in general too faint for spectroscopy with a $4.2 \mathrm{~m}$ telescope. We used this telescope: 1$)$ to corroborate and improve the spectra of both objects $(z=0.24$ and $z=0.39$ ) in the filament; 2) to obtain the redshift and classifications of some other objects in the halo of NGC 7603 (objects like \#17, \#21, \#22 were interesting because of the peculiar position that they occupy with respect the halo and filaments of NGC 7603; (Fig. 1 shows that these sources lie within the halo of NGC 7603); 3) to observe AGN candidates which are not too faint.

Table 4 summarizes the objects crossed by the three positions of the slit and a summary of the analysis of the spectra. Only the intense lines were used to determine the redshift. Figure 4 plots the positions of these slits. The spectra of the filament is poor because the slit in position 1 does not exactly crosses the maximum flux region of the filament, and the width of the slit $(1.2$ arcsec $)$ is small compared with the slitwidth of 5 arcsec used in the observations taken with the
NOT and presented in Paper I ([OIII] detections reported in Fig. 2b of Paper I were spurious). The main spectral features of these objects corrected for redshift and the new ones (objects \#21, \#22, \#23) are shown in Fig. 7. All of them are narrow emission line galaxies.

Table 5 gives the values of the equivalent widths of the different lines. Apart from the errors in the table due to noise in the spectra, these equivalent widths are subject to the possible errors in the subtraction of the sky emission(+filament in objects \#1, \#2). Although the absolute values of $E W$ s can only be taken as a rough approximation, the ratio of close lines is rather exact (because here the uncertainties in the continuum cancel). Roughly, the error would be a factor $\sim 2$ for the continuum in the worst of the cases (assuming the error in the subtraction of the sky+filament is equal to its Poissonian noise), which means that in the worst of the cases the error of $E W$ is a factor two, too.

The spectral classification of narrow emission line galaxies is usually made through the flux ratios of specific lines 


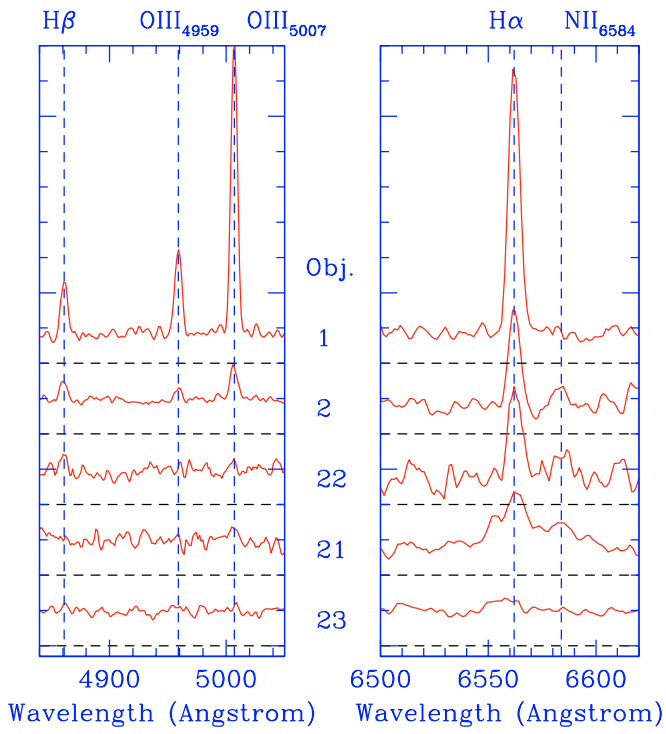

Fig. 7. Main spectral features (corrected for redshift) of objects \#1 $(z=$ 0.245 , in the filament), $\# 2(z=0.394$, in the filament), $\# 21(z=0.117)$, $\# 23(z=0.246)$ and \#22 $(z=0.401)$. Dashed horizontal lines below the spectra are their zero-flux levels. Dashed vertical lines indicate the position of the main spectral features.

corrected of reddening, assuming a constant intrinsic ratio for $\frac{F_{\text {intr. }}(\mathrm{H} \alpha)}{F_{\text {intr. }}(\mathrm{H} \beta)}$. However, this ratio changes when the physical situation in the galaxies does not obey a simple model moreover, we have extinction from the dust in the own galaxy plus the extinction of the filament in cases of \#1 and \#2, and some minor contribution from the Galactic extinction, which are difficult to separate. To classify the galaxies, we use the ratios of lines that are close in wavelength. The difference between the flux ratio and the equivalent width ratio is neglected. The spectral classification criteria is based on the ratios $\log \frac{\mathrm{OIII}_{5007}}{\mathrm{H} \beta}$ and $\log \frac{\mathrm{NII}_{6584}}{\mathrm{H} \alpha}$ (given in Table 5) (Veillux \& Osterbrock 1987; Filippenko \& Terlevich 1992; Dessauges-Zavadsky et al. 2000), and gives the result that the objects in Table 5 are HII-galaxies except object \#21, which might be either a HII-galaxy or a LINER (since its continuum is strong and the emission lines are faint, it might be a "Low Luminosity Active Galactic Nuclei"; Maoz et al. 1998).

\subsection{Galaxies in/behind the filament}

The HII-galaxies embedded in the filament, \#1 and \#2, seem to be indeed quite peculiar star-forming galaxies. The very intensive $\mathrm{H} \alpha$ (equivalent width: $E W(\mathrm{H} \alpha) \approx 80 \AA$ and $160 \AA$ resp.), if correct (i.e. if the continuum is really as low as obtained by us and the sky+filament subtraction has not changed the level of the continuum; roughly, error of $E W$ should be a factor two at most), would be indicative of a vigorously star-formation galaxy. Only $\sim 2 \%$ and $\sim 1 \%$ of the normal HII-galaxies have a so high $E W(\mathrm{H} \alpha)$ (Carter et al. 2001). However, if they were dwarf HII-galaxies, these high $E W$ s would be within the normal expected values. The mean intrinsic colour of these objects is $(B-V)_{0}=0.22 \mathrm{mag}$ and $(B-V)_{0}=0.10 \pm 0.2 \mathrm{resp}$. (with a dispersion of $\approx 0.10 \mathrm{mag}$, plus an error of $\sim 0.15 \mathrm{mag}$ due to the factor 2-error in the value of EW; total: $\sim \pm 0.2 \mathrm{mag}$ ) (Kennicutt et al. 1994, Fig. 2a).

We apply the correction of extinction for the flux of these objects in the following way. First, we derive the observed $(B-V)_{0}$ in the reference system of the galaxy [i.e. we calculate the equivalent $(B-V)$ in the redshifted wavelengths; we do this through the calculation of the flux in the corresponding wavelengths of the redshifted $B$ and $V$ filters given the $U B V R I$ fluxes; this is equivalent to make the k-correction]. These are: $(B-V)_{0}=0.58$ (object \#1) and $(B-V)_{0}=0.45$ (object \#2). We neglect the difference between the colours of a face-on galaxy and other inclinations. Therefore, the differences between these measured colours and the colours expected for these HII-galaxies with the corresponding $E W(\mathrm{H} \alpha+[\mathrm{NII}])$ are: $\Delta(B-V)_{0}=0.48 \pm 0.2$ (object \#1) and $\Delta(B-V)_{0}=0.23 \pm 0.2$ (object \#2). We assume that the measure of the colour has negligible error (the absolute magnitude in each filter has $0.2 \mathrm{mag}$. of error, due to the contamination of the filament, but in the measure of the colour, since the technique to decontaminate the influence of the filament is the same, this error cancels). We attribute these differences to the extinction produced by the filament $\left(z_{\text {fil }}=0.030\right)$ plus the Galactic extinction $(z=0)$ :

$$
\begin{aligned}
\Delta(B-V)_{0}= & A_{\mathrm{Gal} .}\left[\lambda_{B}(1+z)\right]-A_{\mathrm{Gal} .}\left[\lambda_{V}(1+z)\right] \\
& +A_{\text {fil. }}\left[\lambda_{B} \frac{(1+z)}{\left(1+z_{\mathrm{fil}}\right)}\right]-A_{\mathrm{fil} .}\left[\lambda_{V} \frac{(1+z)}{\left(1+z_{\mathrm{fil}}\right)}\right] .
\end{aligned}
$$

The Galactic reddening is relatively low: $\left[A_{\mathrm{Gal}}\left[\lambda_{B}(1+z)\right]-\right.$ $A_{\text {Gal. }}\left[\lambda_{V}(1+z)\right]=0.03$ for both objects according to Schlegel et al. (1998) maps of extinction. Assuming a reddening due to the filament similar to the Galactic dust $\left(A(\lambda) / A_{V}\right.$ from Mathis 1990), we get

$A_{\text {fil.(obj. \#1) }}\left[\lambda^{\prime}=\lambda /\left(1+z_{\text {fil }}\right)\right]=(2.0 \pm 0.9) \frac{A\left(\lambda^{\prime}\right)}{A\left(\lambda_{V}^{\prime}\right)}$,

and

$$
A_{\text {fil.(obj. \#2) }}\left[\lambda^{\prime}=\lambda /\left(1+z_{\text {fil }}\right)\right]=(0.9 \pm 0.9) \frac{A\left(\lambda^{\prime}\right)}{A\left(\lambda_{V}^{\prime}\right)} \text {. }
$$

The corrected magnitudes, including Galactic and filament extinction correction, are given in Table 3.

The calculation of the absolute magnitudes, for instance for the filter $V$, can be carried out by means of:

$M_{V}=m_{\text {corr.ext. }}\left[\lambda_{V}(1+z)\right]-5 \log \left(D_{\mathrm{Mpc}}\right)-25$,

$$
\begin{aligned}
D_{\mathrm{Mpc}}= & \frac{c(1+z)}{H_{0}} \\
& \times \int_{0}^{z}\left[(1+x)^{2}\left(1+\Omega_{M} x\right)-x(2+x) \Omega_{\Lambda}\right]^{-1 / 2} \mathrm{~d} x .
\end{aligned}
$$

We assume $H_{0}=71 \mathrm{~km} / \mathrm{s} / \mathrm{Mpc}, \Omega_{M}=0.27, \Omega_{\Lambda}=0.73$ (derived from WMAP data; Bennett et al. 2003). If we consider the redshifts as indicators of the cosmological distance: $M_{V}($ object $\# 1)=-21.5 \pm 0.8, M_{V}($ object $\# 2)=-18.9 \pm 0.8$. If we considered an anomalous intrinsic redshift case (in such a case, to derive the distance we set $z=z_{\text {fil }}=0.03$ ), the 
Table 5. Equivalent widths (in angstroms) of the emission lines of the five observed narrow emission line galaxies. Errors include a rough determination of the noise and the error in the determination of the continuum, but do not include the error in the subtraction of the sky $(+f i l a m e n t$ in objects \#1, \#2).

\begin{tabular}{llllll}
\hline \hline Line & Object \#1 & Object \#2 & Object \#21 & Object \#22 & Object \#23 \\
\hline $\mathrm{OII}_{3727}$ & $56.3 \pm 6.1$ & $36.2 \pm 7.3$ & $37.1 \pm 9.3$ & $15.3 \pm 2.1$ & $68.4 \pm 75.1$ \\
$\mathrm{NeIII}$ & & $13.0 \pm 3.1$ & & & \\
$\mathrm{H} \beta$ & $13.5 \pm 1.1$ & $43.3 \pm 4.5$ & $3.3 \pm 0.8$ & $6.9 \pm 1.1$ & $3.7 \pm 1.5$ \\
$\mathrm{OIII}$ & & & & \\
$\mathrm{OIII}_{5007}$ & $11.2 \pm 1.2$ & $62.8 \pm 6.3$ & & $2.3 \pm 0.9$ & $3.5 \pm 1.5$ \\
$\mathrm{OI}_{6300}$ & $19.2 \pm 1.7$ & $172.3 \pm 22.1$ & $2.6 \pm 0.5$ & $17.0 \pm 6.1$ & \\
$\mathrm{H} \alpha+\mathrm{NII}_{6548}$ & $81.0 \pm 21.9$ & $160.9 \pm 26.2$ & $15.6 \pm 0.7$ & $25.9 \pm 5.4$ & $18.5 \pm 4.3$ \\
$\mathrm{NII}_{6584}$ & $16.2 \pm 7.3$ & $8.8 \pm 4.6$ & $8.4 \pm 0.5$ & $6.2 \pm 3.1$ & $1.8 \pm 1.8$ \\
$\mathrm{SII}_{6717+6731}$ & & & $7.3 \pm 0.7$ & & \\
\hline $\log \frac{\mathrm{OIII}_{5007}}{\mathrm{H} \beta}$ & $0.15 \pm 0.05$ & $0.60 \pm 0.07$ & $-0.10 \pm 0.13$ & $-0.48 \pm 0.18$ & $-0.02 \pm 0.26$ \\
$\log \frac{\mathrm{NI}_{6584}}{\mathrm{H} \alpha}$ & $-0.70 \pm 0.23$ & $-1.26 \pm 0.24$ & $-0.27 \pm 0.03$ & $-0.62 \pm 0.24$ & $-1.01 \pm 0.45$ \\
\hline
\end{tabular}

results are: $M_{V}\left(\right.$ object\#1) $=-15.2 \pm 0.8, M_{V}($ object\#2 $)=$ $-13.9 \pm 0.8$. In this second case, they would be in the faint tail of the HII-galaxies, type II (Telles \& Terlevich 1995); they would be dwarf galaxies, "tidal dwarfs" as in Stephan's Quintet (Lisenfeld 2002) but with anomalous redshifts, and this would explain the observed strong star formation ratio: objects with low luminosity have higher $E W(\mathrm{H} \alpha)$ (Carter et al. 2001). Teplitz et al. (2003) show examples of compact emission line galaxies with very high $E W(\mathrm{H} \alpha)$ and absolute filterless magnitudes between -14 and -15 (e. g., SPS J082344.12+292351.3).

\subsubsection{Comparison with Paper I and corrections}

With these new spectra, we confirm the redshifts of objects \#1 and \#2 observed in Paper I, and furthermore we detect the $\mathrm{H} \alpha$ emission line in the spectra of both objects. Now it is possible to obtain a more accurate estimation of the linewidth of each object.

The possible classification of both objects as QSOs in Paper I (indeed, we claimed that they were compact emission line objects, either QSOs or HII-galaxies) is not confirmed here. In Paper I, we had not analysed HST data so we could not see whether the objects had any extension. In Fig. 3 of Paper I, we pointed out that the $\mathrm{H} \beta$ line in object \#2 had a $F W H M$ of $49 \AA$ while the forbidden lines had $30 \AA$ in these low resolution spectra; the same was observed for object \#1 with poorer signal/noise. However, in the present WHT-telescope higher resolution spectra, we have not observed this relative broadening, so we think that the apparent excess broadening of $\mathrm{H} \beta$ in Paper I was an artefact due to noise.

In Paper I, we made a rough measurement of the parameter $R_{23}$ directly from ratios of equivalent widths ${ }^{6}$; however, line fluxes corrected for reddening would be necessary. The results

6 There was also an erratum in the reference to Filippenko \& Terlevich (1992) in Paper I: they said that LINERs have $\log _{10} \frac{\mathrm{OIII}_{5007}}{\mathrm{H} \beta} \leq$ 0.5 instead of $\frac{\mathrm{OIII}_{5007}}{\mathrm{H} \beta}>0.5$, and this is used to separate Seyfert $2 \mathrm{~s}$ and LINERS rather than HII-galaxies from LINERs. presented in this paper by making use of $E W \mathrm{~s}$ of close lines give in any case the same conclusion: they are HII galaxies (provided that they have narrow lines).

The slight differences in the $R$-magnitude $(0.2$ and $0.1 \mathrm{mag}$ respectively) with respect to the values presented in Paper I are caused by differences in which the filament was subtracted and are within the errors. In Paper I, it was claimed that the objects have $m_{b_{j}}<21.9$ (corrected for extinction), and this is correct, but for a reason different from the arguments given in Paper I. They are intrinsically blue, but because of the extinction they are observed as red $\left((B-V)_{0}=0.10\right.$ and 0.22 respectively for objects \#1 and \#2 corrected for extinction and the k-correction) instead of blue as claimed in Paper I.

Thus we confirm the main results of Paper I, except for the possible classification of objects \#1 and \#2 as QSOs.

\section{Analysis}

\subsection{A candidate perturber}

According to the results in Sect. 3.1, some close and not very faint neighbour must be in the surroundings of NGC 7603. If we assumed that NGC 7603B and NGC 7603 have the same distance, this filament would be clearly due to the interaction between them. Are there other possible candidates?

There is a galaxy with similar redshift, one magnitude fainter, and 10.3 arcmin from NGC 7603: NGC 7589; or B231533.01-000313.1, three magnitudes fainter and 12.6 arcmin of distance. However, both of them are in the opposite direction of the filament (to the west instead of the east). We do not find any other appropriate candidate for the interaction in the surrounding $30 \mathrm{arcmin}$. Nonetheless, we cannot be sure that this companion object does not exist until we perform spectroscopy of all the surrounding objects around NGC 7603. For instance, galaxy \#29 (see Fig. 4 and Table 2) is four magnitudes fainter than NGC 7603 in the $B$-filter, it has an angular distance of $2.5 \mathrm{arcmin}$ (linear distance larger than $100 \mathrm{kpc}$ ) and it seems to be in the direction of the tail which is extended towards the 
north; it might be a candidate to produce the tidal disturbances. Johnston et al. (2001) in their Eq. (11)/Fig. 6 calculate the expected surface brightness magnitude in such cases. Assuming $t>1$ Gyr, a mass-to-light ratio of 10 , the rotation velocity from NGC 7603 of $200 \mathrm{~km} \mathrm{~s}^{-1}$ in the outer disc, and a distance of the satellite of $100 \mathrm{kpc}$, the observed surface brightness in $R$ of the filament should be $>27 \mathrm{mag} / \operatorname{arcsec}^{2}$; however we observe that it is $24 \mathrm{mag} / \operatorname{arcsec}^{2}$. Therefore, it seems unlikely but remains a possible solution within a standard cosmological redshift hypothesis scenario.

\subsection{Statistics}

\subsubsection{QSO statistics}

In Sect. 3.2, we concluded that there are three objects that follow Eq. (1) within a radius $R=1.5$ arcmin from the center of NGC 7603 (the distance to object \#36). One of these (object \#23) is not a QSO (see Sect. 4). The other two were too faint to be observed spectroscopically. Therefore, we have at most two QSOs in the field of NGC 7603 (other extra QSOs are possible, but with a low probability because the multicolour criterion covers $80-90 \%$ of all QSOs (see Sect. 3.2)) with $m_{b_{j}}=22.6,23.6$ (respectively for objects \#19 and \#36; derived from Sloan filter information as in Sect. 4.1).

The probability for such a event is (assuming roughly that $p_{i}$, the probability for the detection of each QSO, follows $\left.\mathrm{e}^{p_{i}} \sim 1\right)$ :

$P_{Q} \geq \frac{\left(\pi R^{2}\right)^{2} N_{\mathrm{QSO}}\left(m_{b_{j}}<22.6\right) N_{\mathrm{QSO}}\left(m_{b_{j}}<23.6\right)}{2 !}$.

According to the QSO counts from Eq. (A.3), the probability is $P_{Q} \geq 0.029$, so one might expect these background QSOs to occur by chance (2.2- $\sigma$ at most, if both candidates are confirmed as QSOs).

\subsubsection{Probability of NGC 7603 and its 3 companions being a chance projection effect}

From Fig. 1 of Paper I and Figs. 4 or 6 of this paper, it seems extremely improbable that four objects at different distances can show a chance projection in the way these figures reveal. Statistics have been calculated in several ways for some time concerning the anomalous redshift problem (e.g., Arp 1981, 1999a; Burbidge et al. 1997), to assess the probabilities of peculiar configurations. However, many other authors (e.g., Noerdlinger 1975; Sluse et al. 2003) have suspected that these calculations are inappropriate. Some authors also say that one should not carry out a calculation of the probability ("a posteriori probability") for an a priori known configuration of objects (for instance, that they are aligned, or that they form a certain geometrical figure) because all possible configurations are peculiar and unique. The real question is to look for peculiarities associated with peculiar physical representations, not just peculiarities in the sense of being unique.

For our case, we will use a simple fact: the connection of four objects throughout a filament. This aspect represents a physical peculiarity, not because of their uniqueness but because they could be better represented by an alternative theory claiming that the four galaxies are at the same distance, three of them ejected with the filament by the parent galaxy NGC 7603. The question is as follows: what is the probability, $P$, of the apparent fact arising from a random projection of sources with different distances? NGC 7603 has a filament of area $A$, the probability of having three further independent sources, with the corresponding magnitudes of the objects $1-3$, projected on that filament is (assuming that the individual probabilities for each event $p_{i}$ follow $\mathrm{e}^{p_{i}} \approx 1$ ):

$P=\frac{A^{3} N_{1}\left(m \leq m_{1}\right) N_{2}\left(m \leq m_{2}\right) N_{3}\left(m \leq m_{3}\right)}{3 !}$,

where $N_{i}$ is the source density on the sky for the type of sources of object $i$ with apparent magnitude in a given filter (corrected for Galactic+filament extinction) less than $m_{i}$. We use filter $B$, but the statistics would give similar results for any filter. Some authors (e.g., Sluse et al. 2003, hypotheses H2-H3) use in the calculation of the probabilities the limiting magnitude of the survey instead of the magnitude of the object, which gives a higher probability. However, this is not totally correct because, randomly, one would expect most of the detected objects to be close to the limiting magnitude. If this method is followed, the magnitude of the object and the limiting magnitude of the survey are very close and there are no major differences in the calculation; but, if the magnitude of the object is much brighter than the limiting magnitude one should multiply $P$ by a factor that characterizes the probability this object being much brighter than the limiting magnitude (the brighter it is, the lower the probability), and this is equivalent to using the magnitude of the object. So we think that the Sluse et al. (2003) hypotheses H2-H3 are inappropriate.

The area of the filament is approximately 35 arcsec in length multiplied by 4 arcsec in width (the area plotted in Fig. 6):

$A \approx 35^{\prime \prime} \times 4^{\prime \prime}=140 \operatorname{arcsec}^{2}=1.1 \times 10^{-5} \mathrm{deg}^{2}$.

We are not going to use other peculiarities of the system like $i$ / objects \#1 and \#2 are positioned where the filament contacts NGC 7603B and NGC 7603 respectively ii/ objects \#1 and \#2 are HII-galaxies iii/ the distribution of redshifts (from major to minor). If we took these facts into accounts, the probability $P$ would be somewhat lower.

NGC 7603B is a galaxy with $m_{B, 1}=16.6$ (Sharp 1986, corrected only for Galactic extinction; it would be less if the foreground filament produced any extinction in the galaxy). The magnitudes corrected for extinction of the two HII-galaxies are: $m_{B, 2}=21.1 \pm 1.1$ and $m_{B, 3}=22.1 \pm 1.1$. With the counts given by Eq. (A.2), the deduced probability is

$\log P=-8.6 \pm 0.8$.

The error is large, due to the uncertainty of $1.2 \mathrm{mag}$ in the objects \#1 and \#2, but the order of magnitude does not change too much. This means that we have a probability of a few times $10^{-9}$ of finding three galaxies of any type by chance with different distances projected on a filament (an arm or another structure) with an area of $140 \operatorname{arcsec}^{2}$ of an arbitrary galaxy with respective apparent magnitude (corrected for extinction) less than 
or equal 16.6, 21.1, 22.1 respectively, and somewhat higher if the magnitudes are up to 1.2 fainter in the last two objects. If there were no filament extinction at all, the value of $\log P$ would be -7.1 . There are no bias selection effects because we have used complete galaxy counts from complete surveys up to a given magnitude (Appendix A). Certain facts that could make the probabilities calculated above larger are discussed in Sect. 5.3.1.

We could multiply $P$ by the probability of having extremely vigorous star formation in the two HII-galaxies, $P_{2}$. The calculation of $P_{2}$ (with probabilities $\sim 1 \%$ and $\sim 2 \%$ for each galaxy, as said in Sect. 4) is

$P_{2}=0.01 \times 0.02=2 \times 10^{-4}$.

There is near independence between both probabilities, so the global probability is the product of $P$ and $P_{2}$. There is a correlation between absolute magnitudes and the star formation ratio: the fainter the HII-galaxy, the higher is the star formation ratio (Carter et al. 2001). Teplitz et al. (2003) find in their sample that many galaxies have large values of $E W(\mathrm{H} \alpha)$ but their objects are in general intrinsically fainter. However, if we accept the cosmological redshift hypothesis, our two HII-galaxies would have average or high luminosities (absolute magnitudes in $V$ from -19 to -22 ); therefore, it is not possible to have an enhancement of the probability due to some selection effect (neither the Malmquist bias nor the opposite one). We will not consider the low value of $P_{2}$ until we can have more accurate measures of $E W \mathrm{~s}$, and we will only discuss the probability $P$ quoted above.

According to SIMBAD there are 237 AGN-galaxies in all the sky with a $B$ magnitude less than 14.0 (the magnitude of NGC 7603; de Vaucouleurs et al. 1991). Therefore, the probability to have an AGN with a $B$ magnitude up to 14.0 with the cluster of coincidences that we observe in NGC 7603 is:

$P_{\text {all AGNs }, m_{B}<14.0} \sim 7 \times 10^{-7}$.

Considering all the galaxies independently of whether they are AGNs or not, according to SIMBAD there are 3655 galaxies with $m_{B}<14.0$, so the probability to have the observed configuration around NGC 7603 would be:

$P_{\text {all galaxies, } m_{B}<14.0} \sim 10^{-5}$.

This assumes that all the galaxies have some filament/arm like NGC7603 to find the background objects, which is not necessarily the case, so we are overestimating the probabilities. These probabilities are calculated assuming that there is only one case with this cluster of chance circumstances. However, cases like 3C 212 (Stockton \& Ridgway 1998), NGC 3067 (Carilli et al. 1989; Carilli \& van Gorkom 1992), NGC 3628 (Arp et al. 2002); NGC 1232, NGC 4151 or NGC 622 (Arp 1987) or the cases mentioned in Arp (1980) also present some filaments/arms that have at their ends, or somewhat beyond the end in the direction of the filament, some galaxy or QSO with different redshift (Note: many of these examples have however magnitude large than 14.0 in B). Another possible example is the tail between NGC 7320 and NGC 7318 in Stephan's Quintet (Moles et al. 1998; Gutiérrez et al. 2002;
Williams et al. 2002). In some of these tails the presence of dwarf galaxies looking like objects 1 and 2 has been discovered even at relatively large distances from the disrupted galaxy (Gallagher et al. 2001) so, again, we are overestimating the probability.

It is remarkable that the presence of the filament gives the configuration a low probability, but even without taking into account the presence of the filament, the probability is low. Given a square of diagonal 59 arcsec (the separation between NGC 7603 and NGC 7603B), the chance of having four galaxies with magnitudes in the $B$-filter of 13.8 (the magnitude of NGC 7603 corrected for extinction), 16.6, 21.1 and 22.1 would be $3.5 \times 10^{-11}$ (again with Poissonian statistics and the counts given by Eq. (A.3)). There are $3.2 \times 10^{8}$ squares like this on the whole sky, so the probability of finding only one square in the whole sky with this congregation of four objects of different distances is $1.1 \times 10^{-2}$.

\subsection{Possible explanations for the low probability observed configuration}

Possible scenarios to explain the present case of NGC 7603 depend on the possible explanations for the redshift of the objects (Narlikar 1989; Hoyle \& Burbidge 1996): cosmological (with the observed configuration due to clusters in the line of sight, or gravitational lensing), Doppler, gravitational or others.

\subsubsection{Clusters in the line of sight?}

If we have found a line of sight with many clusters of galaxies, that would increase significantly the density of sources with respect to a Poissonian distribution. However, a configuration in which our line of sight crosses three clusters of galaxies at different redshifts $(z=0.056, z=0.245$ and $z=0.394)$ is not justified because the increase in the probability due to the increase of the density in lines of sight with clusters is compensated for by the additional factor to be multiplied by $P$ to take into account the probability of finding clusters in the line of sight. On average, in all the arbitrary lines of sight of the sky, the probability will be given by the above value of $P$.

Let us assume that the clusters in the sky have the same size, $A_{\mathrm{c}}$, a Poissonian distribution, and the same number of galaxies up to a given magnitude, $n_{\mathrm{c}}$ (galaxies/cluster). This is a very rough model, because it is clear that $A_{\mathrm{c}}$ and $n_{\mathrm{c}}$ depend on the redshift; however, for our present arguments, the estimation with mean values of $A_{\mathrm{c}}$ and $n_{\mathrm{c}}$ is enough. In such a case, the total number of galaxies, $N$, is:

$N=N_{f}+N_{\mathrm{c}} n_{\mathrm{c}}$

where $N_{\mathrm{f}}$ is the density of field galaxies (galaxies/ $/ \mathrm{deg}^{2}$ ) and $N_{\mathrm{c}}$ is the density of clusters (clusters/ $/ \mathrm{deg}^{2}$ ). An example of a probability calculation would be the one to have three galaxies belonging to three different clusters in the area $A$ (we assume that they have the same magnitude, for a simplistic calculation, although it can be generalized to any magnitude distribution), i.e. the probability of three clusters being in the line of sight multiplied by the probability of three galaxies from different clusters 
being in the area $A$ of the filament given the density of galaxies in a cluster,

$$
\begin{aligned}
P & =\frac{\left(N_{\mathrm{c}} A_{\mathrm{c}}\right)^{3}}{3 !} \frac{\left(A \frac{n_{\mathrm{c}}}{A_{\mathrm{c}}}\right)^{3}}{3 !} \\
& =\frac{A^{3} N^{3}}{3 !}\left(1-\frac{N_{\mathrm{f}}}{N}\right)^{3} \frac{1}{3 !}<\frac{A^{3} N^{3}}{3 !}
\end{aligned}
$$

that is, the probability is lower than $\frac{A^{3} N^{3}}{3 !}$, which is the probability we calculated in (8). Therefore, the supposition of a line of sight with three clusters would make the probability smaller instead of larger, and similarly for a lower number of clusters.

Indeed, it is not likely to find clusters of galaxies at $z=$ 0.245 and $z=0.391$, in spite of the two pairs of HII-galaxies with close redshifts, because HII-galaxies are much less common in clusters than in field galaxies (Gisler 1978; Dressler et al. 1985; Biviano et al. 1997).

Nonetheless, although the low probabilities cannot be justified by this scenario of clusters, and although the high star formation ratios seem to point in the opposite direction, we also have object \#23 with nearly the same redshift as object \#2, and object \#22 with a difference of 0.007 in redshift with respect to object \#1. Perhaps they form small groups of galaxies with separations of 0.5 or $2 \mathrm{Mpc}$ (for the pairs at $z=0.25$ and $z=0.40$ respectively).

We have considered above that the distribution of clusters is Poissonian; it might be that we have detected two or three clusters in the line of sight for some special reason. Could our line of sight be tangential to a wall or sheet within the large scale structure, for instance? This seems difficult to imagine, since we would need a wall of size $2 \mathrm{Gpc}$. The Hydro-Gravitational Theory (Gibson 1996; Gibson \& Schild 2003) would claim that the members of a cluster (NGC 7603, NGC 7603B, object \#1 and object \#2) formed together, and that they remained together until the uniform expansion of space in the universe finally overcame the gravitational and frictional forces of the cluster, and the different galaxies separated with very small transverse velocity with respect to the line of sight because of the halo gas friction and their sticky beginning. The stretching would be along a pencil beam of length $\sim 2 \mathrm{Gpc}$ in the line of sight by the expansion of the universe, but a preferred direction of the expansion instead of an isotropic expansion is not justified.

\subsubsection{Gravitational lensing}

A better explanation might in principle be found if we considered some kind of gravitational lensing. For instance, amplifications up to a factor $\sim 30$ are expected (Ellis et al. 2001) for background objects apparently close to the central parts of massive clusters. The effect produced by an individual galaxy like NGC 7603 should be much smaller, and the low redshift galaxy $(z=0.029)$ NGC 7603 , as the putative lens of very distant sources $(z=0.245$ and $z=0.394)$ would have a very small amplification because of the large angular distance of the sources. Given a galaxy with Einstein radius $\theta_{\mathrm{E}}$, the enhancement in the density of background objects as a function to the angular distance, $\theta$, to this galaxy will be (Wu 1996):

$q_{Q}(\theta)=\frac{N\left[m<m_{b, \text { lim }}+2.5 \log \mu(\theta)\right]}{N\left(m<m_{b, \text { lim }}\right)} \frac{1}{\mu(\theta)}$,

where $\mu$ is the magnification factor (Wu 1996),

$\mu(\theta) \approx \frac{\theta}{\theta-\theta_{\mathrm{E}}}$.

To have a value of $P$ that is not very low, we would need this to be $\sim 10^{3}-10^{6}$ higher, i.e. an average enhancement of $\sim 10-100$ in density for each of the galaxies. With the counts of Eq. (A.2), for the lowest enhancement, this requires an average magnification of $\mu(\theta)$ of $\sim 2 \times 10^{4}$. We would need to be in the ring $\theta_{\mathrm{E}}<\theta<\theta_{\mathrm{E}}\left(1+5 \times 10^{-5}\right)$, which is very narrow with a very low probability; so again the problem is not solved by this artefact. It is clear from Eq. (16) that the density of sources does not increase so quickly unless the counts increase extremely quickly with the limiting magnitude, which is not the case. The enhancement in the source counts increases because of the flux increase of each source but decreases because of the area distortion, which reduces the number counts by losing the sources within a given area (Wu 1996).

In our case, since the distance of the sources to the centre of NGC 7603 is $0.5-1$ arcmin, we would need either a very large value of the Einstein radius of the gravitational lens placed in the centre of NGC 7603, which would require a huge mass (for instance, an average $\mathrm{E} / \mathrm{S} 0$ galaxy has a $\theta_{\mathrm{E}} \approx 1.33 \mathrm{arcsec}$, Wu 1996), or that the gravitational lenses be not so massive but much closer to the magnified objects. The first possibility may be automatically rejected, since even in the case that NGC 7603 had the mass of a cluster of galaxies, the magnification would affect at most only one of the three objects in the filament, the one closer to its Einstein radius. The second hypothesis, the possibility that multiple minilenses are distributed in the halo of the galaxy, has already been proposed: gravitational mesolensing by King objects (Baryshev \& Bukhmastova 1997; Bukhmastova 2003). The strong gravitational lensing would be produced by King lenses: globular clusters (Bukhmastova 2003), dwarf galaxies, or clusters of hidden mass with masses between $10^{3}$ and $10^{9} M_{\odot}$. This is an interesting idea, although we are not convinced by the proof presented by one of authors of the idea (Bukhmastova 2001) which reveals excesses of pairs of galaxy/QSO with $z_{\mathrm{gal}}>0.9 z_{\mathrm{QSO}}$, because many of these pairs were indeed the same object classified both as QSOs and galaxies. Nevertheless, in our particular case, it does not solve the low probability $P$, because only in narrow rings is the enhancement high enough, and these narrow rings have a very small area, so, the probability of these being a large number of sources is small.

\subsubsection{Non-cosmological redshift hypotheses}

The relative angular configuration of NGC 7603, NGC 7603B, object \#1 and \#2, the filament connecting all of them and the probability that two of two HII-galaxies in the filament have very high star formation ratios, if we accepted as valid the measures of the EWs, could be explained as a consequence 
of a physical interaction between them. An interpretation that explains the configuration as equivalent to other systems in interaction would be clearly preferred over one in which the configuration is purely a projection effect according to the calculations in Sect. 5.2.2. In that case, the filament would be a sign of disruption in NGC 7603 owing to the proximity of NGC 7603B. This is reinforced by the fact that both NGC 7603 and NGC 7603B show asymmetries in the halo. The narrow emission line galaxies \#21, \#22, \#23 on the other side of NGC 7603 might also be embedded in the extension of the halo pointing to these objects.

In such a case, the redshifts would be non-cosmological. Some of the possible explanations for an intrinsic redshift with standard physics are now discussed:

Doppler: Considering only the system of NGC 7603 and NGC 7603B, which has a difference of around $8000 \mathrm{~km} \mathrm{~s}^{-1}$ is it possible that both galaxies are at the same cosmological distance and that the difference in redshift reflects a difference in peculiar velocities? The known examples of interacting galaxies in the field show differences in velocity between them $\sim 1000 \mathrm{~km} \mathrm{~s}^{-1}$. The higher density of objects in a cluster of galaxies and the dispersion of velocities within them could favour high speed collisions with differences in velocity between the interacting galaxies of a few $\sim 1000 \mathrm{~km} \mathrm{~s}^{-1}$. The possibility of an encounter between groups of galaxies with a difference in velocity of $\sim 4000 \mathrm{~km} \mathrm{~s}^{-1}$ has been considered by de Ruiter et al. (1998) as a possible explanation of the peculiar field around B2 1637+29. However, as far as we know, no example of such a collision in the field with a difference in velocity as large as that existing between NGC 7603 and NGC 7603B has been reported so far, and it would be unexplained in the framework of models of galaxy formation. Furthermore, the extremely high velocity differences of the HII-galaxies would disrupt the system quickly and there would be cases of blueshifts (in this or other anomalous redshift cases).

Gravitational: Anomalous redshifts could alternatively be explained in terms of highly collapsed matter (Narlikar 1989). The gravitational redshift explanation could then be an explanation in terms of standard physics although we would need either very high masses or very low radii for these objects. High mass seems to be excluded since this would affect the rotation curves in the QSO-galaxy pairs (Hoyle \& Burbidge 1996). Very dense non-high mass objects could explain the situation, but the HII-galaxies and NGC 7603B are extended objects; unless most of the mass is concentrated in the very centre of the nucleus, giving an intrinsic redshift, and the outer part of the galaxies have normal cosmological redshifts. At present, we have not detected these differences of redshifts within the HII-galaxies, and this possibility can be discarded for NGC 7603B.

Multiple scattering: Dynamic multiple scattering has been also proposed to explain these systems. Results in statistical optics (Wolf 1986; Datta et al. 1998a,b) show that a shift in the frequency of spectral lines is produced with redshift independent of the frequency when the light passes through a turbulent (or inhomogeneous) medium, because of multiple scattering effects (Roy et al. 2000). The anomalies could be caused by certain special conditions in the surroundings of the anomalous redshift objects. The scattering solution has been proposed as a way to explain the loss of energy of the photons ("the tired light theory"), an alternative to the cosmological redshift. There were several proposals in terms of photon-photon or photon-matter interaction due to some quantum effects (e.g., Finley-Freundliech 1954; Pecker et al. 1972; Laio et al. 1997). Potentially, this effect could explain the high redshifts of some anomalous redshift objects, since light travelling through their outer atmospheres could be redshifted, and the blurring would not be a problem here since the distance travelled is short.

\subsubsection{Variable mass hypothesis}

Non-standard physics has also been used to explain the redshift problem. Hoyle \& Narlikar (1964) developed a new theory of gravitation with particle masses depending on time according to $m \sim t^{2}$ and redshifts

$1+z=\frac{\lambda_{\text {source }}}{\lambda_{\text {observer }}}=\frac{m_{\text {observer }}}{m_{\text {source }}}$

where "observer" and "source" stands for the measures from the different system at the Earth and in the source respectively. The explanation that these authors give for anomalous redshift galaxies is that new matter is being created there with $t=0$, $m_{\text {source }}=m(t=0)$ for the new matter and that the mass varies with the age (Narlikar 1977; Narlikar \& Arp 1993) to produce different redshifts.

\subsubsection{Higher redshift galaxies ejected by a parent galaxy?}

Some proposed models (e.g. Arp 1999a,b; Arp \& Russell 2001; Burbidge 1999b; Bell 2002a,b) assume that some QSOs are ejected by a parent galaxy and decrease in redshift as they move outward, often along the minor axis (the more recent ejections are near the axes, but they later move away because of peculiar motions, precession of the galaxy or the spin axis of the nucleus; Arp 1999b), until they reach a maximum distance of $\sim 500 \mathrm{kpc}$ when they fall back to the parent galaxy and turn into compact, active galaxies and, when they are older, into normal galaxies. Galaxies would beget galaxies; they would not be made from initial density fluctuations in a Big Bang Universe (Burbidge 1999b). It is usually claimed that the variable mass hypothesis is the explanation for the intrinsic redshifts. However, the scenario of "galaxies beget galaxies (with different redshift)" should be considered as a separate matter from the variable mass hypothesis or the Quasi Steady State Theory because other explanations of the redshifts and other cosmological scenarios could be compatible with the present idea.

This scenario seems to fit the observed system quite well: we would have three (or possibly only two or one, if we considered that only some of the objects are background galaxies) ejected together with the material of the filament, and any of the objects \#21, \#22 or \#23 might be part of the ejection on the 
other side of the galaxy, or the QSO candidates whose spectra remains to be taken (\#19,\#36). The near coincidence of the redshifts of two of these objects with the redshifts of the HII galaxies in the filament suggests that they have a common interpretation: either objects with $z=0.245$ and $z=0.246$ and objects with $z=0.394$ and $z=0.401$ belong to the same two groups of galaxies (in a cosmological redshift interpretation) or all of them are ejected by the parent galaxy NGC 7603 (in a non-cosmological redshift interpretation). Nevertheless in the cosmological interpretation we still need to explain the low value of $P$. Therefore, if we want to avoid the word "coincidence" in all aspects (positions and redshifts) we must assume that all objects $(\# 1, \# 2, \# 22, \# 23)$ are ejected by the parent galaxy.

HST images might show the interaction of the filament with objects \#1 and \#2 (see Sect. 3.2.1). The narrow line character in these objects (in principle, classified as HII galaxies according to their line ratios) would be a result of the ejection and interaction with the filament. Evidence is shown in other papers (e.g., Keel et al. 1998, 1999; Arp 1999a; Burbidge et al. 2003) that when QSOs interact with ambient material they become less compact and have narrower lines emitted from more a more diffuse body. This could be the physical explanation. Dynamically disturbed starburst galaxies, as illustrated by the case of NGC 2777 (Arp 1988), tend to be the small companions of larger nearby galaxies belonging to older stellar populations. According to Arp (1988), they are recently created galaxies in which star formation is stimulated by recent ejection from the parent galaxy; some older stars, together with stellar material, are suggested to be removed from the larger galaxy in the course of this ejection. In the system NEQ3 near NGC4151, a QSO and an HII-galaxy have almost identical redshifts, with a separation of 2.8 arcsec and nearly the same magnitudes; the HII-galaxy is embedded in a filament while the QSO is a little bit further away (Gutiérrez \& López-Corredoira 2004). It is another example of environment where QSOs and narrow emission line galaxies have some relation. The interaction between them could also explain the high observed equivalent width in their $\mathrm{H} \alpha$ lines.

According to this theory, the intrinsic redshifts are indicated to evolve in discrete steps as the QSOs evolve into galaxies (Arp 1999b). The peaks in the quantization of the redshifts would be at redshifts around 0.06, 0.30, 0.60, 0.96, 1.41, 1.96 (Arp et al. 1990; Burbidge \& Napier 2001), although the dispersion is large mainly because of peculiar velocities.

The redshifts of the HII-galaxies suggest a possible relation in pairs of objects: the pair in the filament with redshifts 0.245 and 0.394 could stem from the same original source with intrinsic redshift $\approx 0.32$ (the exact value of this number indeed depends on the respective masses of the HIIgalaxies), and a superposed Doppler radial velocities of around $\pm 17000 \mathrm{~km} \mathrm{~s}^{-1}$, for instance (velocities of this order are obtained by Bell 2002a). A similar pair might be the HII-galaxies at 0.246 and 0.401 away from the filament, on the other side of NGC 7603, but these might be in the background. This value of $z \approx 0.32$ (around $z \approx 0.28$ for an observer at NGC 7603) is close to the peak in the periodicity of QSOs/galaxies of $z=0.30$ (Arp et al. 1990; Burbidge \& Napier 2001). The same argument might be applicable to the pair of objects \#22, \#23. The emission in pairs or triplets could be very common according to this theory. Bell (2002a,b) proposes that the ejection occurs in triplets along the rotation axis of the central torus, and that these triplets are composed of a singlet and a pair that simultaneously separate in opposite directions and at $90^{\circ}$ to the triplet ejection direction. The separation between the singlet and the pair is higher than the pair separation; if this were the case in NGC 7603, we would have to find the singlets somewhere in the field of NGC 7603.

We do not have enough information about the distances of the sources with respect to the parent galaxy to build an unique 3-D representation. For instance, Fig. 8 represents a possible configuration according to the ejection theory. The inclination of the galaxy is around 20 degrees with respect the line of sight (ellipticity $\approx 0.35$ ), so slight deviations of the objects from the rotation axis could produce the projected image that we have observed. Figure 8 shows a model in which the filament is not in the plane of the galaxy, but is ejected in a direction nearly perpendicular to the plane.

The filament does not have a blue colour like the other spiral arms in NGC 7603; neither does it have young star formation since it has no emission lines (paper I); instead, it has a red colour (see Fig. 3), like the old population of the disc of NGC 7603. Therefore, the filament could possibly be some material stripped from the main galaxy as a result of some tidal interactions or ejection. A reason for the visibility of the filament in this ejection with $24.0 \mathrm{mag} / \operatorname{arcsec}^{2}$, while is not observed in other systems, might be the integration along the line of sight of a filament that is nearly tangential to the line of sight and provides a high column density. Nonetheless, there are some other cases that also have similar continuous or nearly-continuous filaments/arms (with some gaps) connecting different-redshift objects (see Sect. 5.2.2). NGC 7603 is perhaps the clearest case, but it may not be unique.

The other side of NGC 7603 (behind NGC 7603 if we assume that the filament and its ejected objects are in front of it) could also have some ejected objects. We do not see the filament there, possibly because it is behind the galaxy.

Other possible scenario within this ejection hypothesis would be that all the galaxies are in the plane of NGC 7603 . It is noteworthy that all the five HII galaxies and NGC 7603B are almost aligned, which could lead us to think of an ejection along some common axis. However, this axis would not be the rotation axis, which is the expected axis in ejection theories.

\section{Summary}

- We present new observations in the field of the Seyfert galaxy NGC 7603. These comprise broad and narrow band imaging, and intermediate resolution spectroscopy of several objects in the field.

- We have delineated the halo around NGC 7603 out to the isophote $26.2 \mathrm{mag} / \operatorname{arcsec}^{2}$ in the Sloan $r$ band filter finding several signs of irregularities and asymmetries towards the east. Neither these eastern asymmetries nor the filament towards the east, apparently connecting NGC 7603 and NGC 7603B, can be easily understood in an isolated 


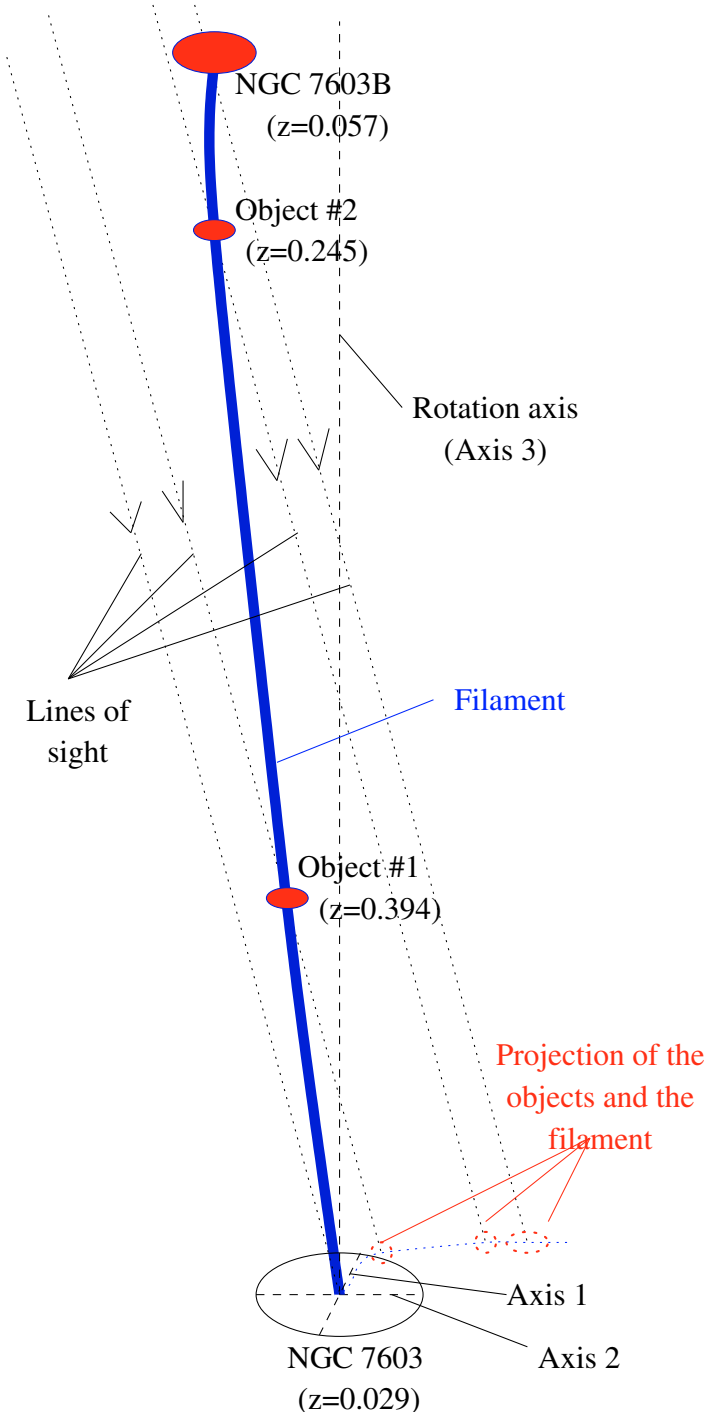

Fig. 8. Possible representation of the system of NGC 7603/NGC 7603B/Object \#1/Object \#2 if we accept the hypothesis of the three last objects ejected by the parent galaxy, NGC 7603. The inclination of NGC 7603 with respect the line of sight is $\approx 20^{\circ}$. The major axis in the projected image (Axis 1 ) has a position angle $\approx-15^{\circ}$; the minor axis in the projected image is "Axis 2".

galaxy, and until now no good candidates for companions on the east side of NGC 7603 with the same redshift have been found.

- With improved spectra with respect to those published in Paper I, we have confirmed the redshift of the two objects in the filament connecting NGC 7603 and NGC 7603B and we have observed their $\mathrm{H} \alpha$ lines for the first time.

- The better resolution achieved in these new spectra and HST imaging of these objects have allowed their more accurate classification as HII galaxies. We have not detected any signs of variability in these objects at levels $\geq 0.3-0.4 \mathrm{mag}$. We found very strong star formation (or whatever the cause of the high equivalent widths of $\mathrm{H} \alpha$ lines) in both of them and the HST images show some distortions in the shape of both galaxies, which might suggest an interaction with the filament.

- We have detected new narrow emission line galaxies at $z=0.246,0.117$ and $0.401,0.8,1.5,1.7 \mathrm{~min}$ to the west of the filament between NGC 7603 and NGC 7603B. The nearly coincidence of the redshifts of two of these objects with the redshifts of the HII galaxies in the filament make us think that they have likely a common interpretation: either objects with $z=0.245$ and $z=0.246$ and objects with $z=0.394$ and $z=0.401$ belong to the same two groups of galaxies respectively (in a cosmological redshift interpretation) or all of them are ejected by the parent galaxy NGC 7603 (in a non-cosmological redshift interpretation).

- The probability of a fortuitous accumulation of objects as bright as NGC 7603, NGC 7603B, and the two objects in the filament is $\sim 3 \times 10^{-9}$. The detection of vigorous star formation observed in the HII-galaxies of the filament, if confirmed, would have a probability $2 \times 10^{-4}$, giving a total probability $\sim 6 \times 10^{-13}$. They look like dwarf HII-galaxies (non-cosmological redshift) rather than normal/giant HII-galaxies (cosmological redshift).

- An explanation in terms of cosmological redshifts (with or without gravitational lensing, with or without clusters in the line of sight has a very low probability although it is not impossible. Alternative explanations have been analysed.

Acknowledgements. Thanks are given to the referee Jack Sulentic for useful comments and criticisms on the interpretations of probabilities. We thank also to Evencio Mediavilla (IAC) who read the manuscript and gave helpful comments. This research has made use of the SIMBAD database, operated at CDS, Strasbourg, France.

\section{Appendix A: Cumulative counts of galaxies and QSOs}

The cumulative counts of galaxies in the $B$-band can be derived from differential galaxy counts from Metcalfe et al. (1991) for galaxies between $20.5<B<24.5$ (magnitudes corrected for extinction):

$\log N\left(B_{0}-0.25<B<B_{0}+0.25\right)=0.494 B_{0}-7.72 \mathrm{deg}^{-2}$. (A.1)

The cumulative count is:

$$
\begin{aligned}
N\left(B<B_{\text {lim }}\right) & \approx 2 \int_{-\infty}^{B_{\lim }} \mathrm{d} B_{0} N\left(B_{0}-0.25<B<B_{0}+0.25\right) \\
& =3.35 \times 10^{-8} \times 3.12^{B_{\lim }} \mathrm{deg}^{-2}
\end{aligned}
$$

This assumes as an approximation that Eq. (A.1) applies for $B_{0}<20.5$, which is more or less correct because the change of slope is very small for lower magnitudes.

The cumulative QSO counts are given by:

$N\left(b_{j}<b_{j, \lim }\right) \approx 1981-214.2 b_{j, \lim }+5.792 b_{j, \mathrm{lim}}^{2} \mathrm{deg}^{-2}$.

We derived this expression by fitting the cumulative QSO counts in the photographic $b_{j}$ filter from the survey by Boyle et al. (2000), based in a multicolour selection of QSOs. Figure A.1 shows that fit. The Boyle et al. (2000) data are for $b_{j}<21.0$, but we extrapolate the fit (A.3) as an approximation 


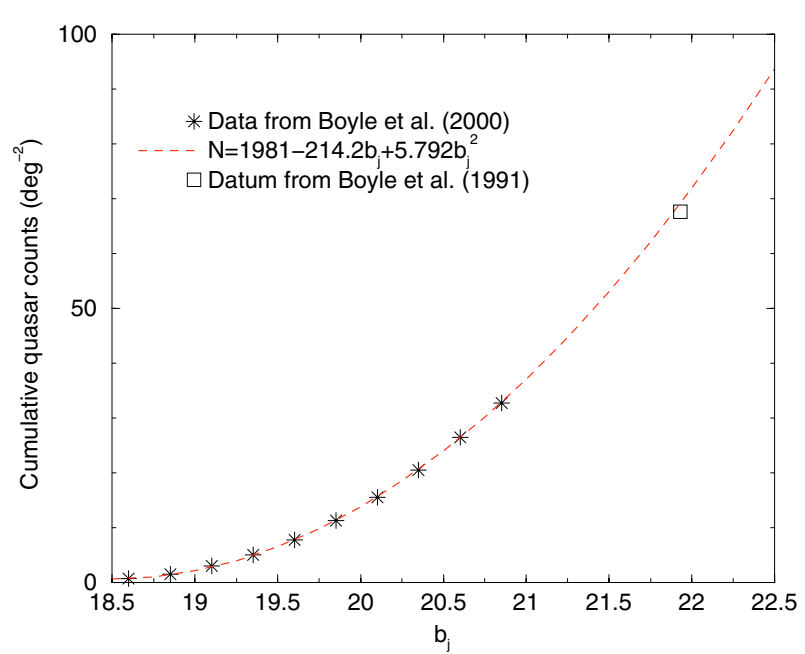

Fig. A.1. Cumulative QSO counts data (Boyle et al. 2000; 1991) and a fit of a second polynomial degree to the Boyle et al. (2000) data.

to higher magnitudes. Another point obtained from a spectroscopic survey of faint QSOs (Boyle et al. 1991) confirms that the fit and its extrapolation are reasonably good (see Fig. A.1). The uncertainties are low: the multicolour selection of QSOs is complete for QSOs of redshift of $z<2.2$ (90\%), or $80 \%$ for $z>2.2$ (Boyle et al. 2000; Meyer et al. 2001).

\section{References}

Arp, H. C. 1971, ApL, 7, 221

Arp, H. C. 1975, PASP, 87, 545

Arp, H. C. 1980, ApJ, 239, 469

Arp, H. C. 1981, ApJ, 250, 31

Arp, H. C. 1987, QSOs, Redshifts and Controversies (Berkeley: Interstellar Media)

Arp, H. C. 1988, in High energy astrophysics: Supernovae, remnants, active galaxies, cosmology (Berlin: Springer-Verlag), 160

Arp, H. C. 1998, Seeing red: redshifts, cosmology and academic science (Montreal: Apeiron)

Arp, H. C. 1999a, ApJ, 525, 594

Arp, H. C. 1999b, Active Galactic Nuclei and Related Phenomena, ed. Y. Terzian, E. Khachikian, \& D. Weedman (S. Francisco: ASP), 347

Arp, H. C., \& Russell, D. A. 2001, ApJ, 549, 802

Arp, H. C., Bi, H., Chu, Y., \& Zhu, X. 1990, A\&A, 239, 33

Arp, H. C., Burbidge, E. M., Chu, Y., \& Zhu, X. 2001, ApJ, 553, L11

Arp, H. C., Burbidge, E. M., Chu, Y., et al. 2002, A\&A, 391, 833

Baryshev, Y. V., \& Bukhmastova, Y. L. 1997, Astron. Rep., 41, 436

Bell, M. B. 2002a, ApJ, 566, 705

Bell, M. B. 2002b, ApJ, 567, 801

Benítez, N., Sanz, J. L., \& Martínez-González, E. 2001, MNRAS, 320, 241

Bennett, C. L., Halpern, M., Hinshaw, G., et al. 2003, ApJS, 148, 1 Bertin, E., \& Arnouts, S. 1996, A\&AS, 117, 393

Biviano, A., Katgert, P., Mazure, A., et al. 1997, A\&A, 321, 84

Boyle, B. J., Jones, L. R., \& Shanks, T. 1991, MNRAS, 251, 482

Boyle, B. J., Shanks, T., Croom, S. M., et al. 2000, MNRAS, 317, 1014

Bukhmastova, Y. L. 2001, Astron. Rep., 45, 675

Bukhmastova, Y. L. 2003, Astron. Lett., 29, 214

Burbidge, G. R. 1996, A\&A, 309, 9

Burbidge, G. R. 1999a, ApJ, 511, L9
Burbidge, G. R. 1999b, in Cosmological Parameters and the Evolution of the Universe, ed. K. Sato (Dordrecht: Kluwer), 286

Burbidge, G. R. 2001, PASP, 113, 899

Burbidge, E. M., Burbidge, G. R., Arp, H. C., \& Zibetti, S. 2003, ApJ, 591,690

Burbidge, G. R., Hoyle, F., \& Schneider, P. 1997, A\&A, 320, 8

Burbidge, G. R., \& Napier, W. 2001, AJ, 121, 21

Carilli, C. L., \& van Gorkom, J. H. 1992, ApJ, 399, 373

Carilli, C. L., van Gorkom, J. H., \& Stocke, J. T. 1989, Nature, 338, 134

Carter, B. J., Fabricant, D. G., Geller, M. J., \& Kurtz, M. J. 2001, ApJ, 559,606

Croom, S. M., Smith, R. J., Boyle, B. J., et al. 2001, MNRAS, 322, L29

Datta, S., Roy, M., \& Moles, M. 1998a, Inst. Journ. Theor. Phys., 37, 1313

Datta, S., Roy, M., \& Moles, M. 1998b, Inst. Journ. Theor. Phys., 37, 1469

de Ruiter, H. R., Fan ti, R., Parma, P., et al. 1998, MNRAS, 337, 711

Dessauges-Zavadsky, M., Pindao, M., Maeder, A., \& Kunth, D. 2000, A\&A, 355, 89

Dressler, A., Thompson, I. B., \& Shectman, S. A. 1985, ApJ, 288, 481

de Vaucouleurs, G., de Vaucouleurs, A., Corwin JR., H. G., et al. 1991, Third Reference Catalogue of Bright Galaxies (New York: Springer-Verlag)

Ellis, R., Santos, M. R., Kneib, J. P., \& Kuijken, K. 2001, ApJ, 560, L119

Filippenko, V. A., \& Terlevich, R. J. 1992, ApJ, 397, L79

Finley-Freundlich, E. 1954, Phil. Mag., 45, 303

Gallagher, S. C., Charlton, J. C., Hunsberger, S. D., et al. 2001, AJ, 122,163

Gaztañaga, E. 2003, ApJ, 589, 82

Gibson, C. H. 1996, Appl. Mech. Rev., 49, 299

Gibson, C. H., \& Schild, R. E. 2003, AJ, submitted, preprint [arXiv: astro-ph/0304107]

Gisler, G. R. 1978, MNRAS, 183, 633

Goodrich, R. W. 1989, ApJ, 340, 190

Gott, J. R., III, \& Gunn 1974, ApJ, 190, L105

Gutiérrez, C. M., \& López-Corredoira, M. 2004, ApJ, 605, L5

Gutiérrez, C. M., López-Corredoira, M., Prada, F., \& Eliche M. C. 2002, ApJ, 579, 592

Hickson, P. 1982, ApJ, 255, 382

Hoyle, F. 1972, Henry Norris Russel Lecture, The Seattle Meating of the A\&AS

Hoyle, F., \& Burbidge, G. R. 1996, A\&A, 309, 335

Hoyle, F., Burbidge, G., \& Narlikar, J. V. 1993, ApJ, 410, 437

Hoyle, F., \& Narlikar, J. V. 1964, Proy. Roy. Soc. London A, 282, 191

Jain, B., Scranton, R., \& Sheth, R. K. 2003, MNRAS, 345, 62

Johnston, K. V., Sackett, P. D., \& Bullock, J. S. 2001, ApJ, 557, 137

Keel, W. C., Cohen, S. H., Windhorst, R. A., \& Waddington, I. 1999, AJ, 118, 2547

Keel, W. C., Windhorst, R. A., Cohen, S. H., Pascarelle, S., \& Holmes, M. 1998, NOAO Newsletter, 53, 1

Kennicutt, R. C. Jr., Tamblyn, P., \& Congdon, C. W. 1994, ApJ, 435, 22

Kollatschny, W., Bischoff, K., \& Dietrich, M. 2000, A\&A, 361, 901

Kondratko, P. T., Greenhill, L. J., \& Moran, J. M. 2001, A\&AS, 199, 5101

Kopylov, I. M., Lipovetskii, V. A., Pronik, V. I., \& Chuvaev, K. K. 1974, Afz, 10, 483

Laio, A., Rizzi, G., \& Tartaglia, A. 1997, Phys. Rev. E, 55, 7457

Landolt, A. U. 1992, A\&A, 104, 340

Lisenfeld, U., Braine, J., Duc, P.-A., et al. 2002, A\&A, 394, 823 
López-Corredoira, M., \& Gutiérrez, C. M. 2002, A\&A, 390, L15 (Paper I)

Malkan, M. A., Gorjian, V., \& Tam, R. 1998, ApJS, 117, 25

Maoz, D., Koratkar, A., Shields, J. C., et al. 1998, AJ, 116, 55

Mathis, J. S. 1990, ARA\&A, 28, 37

Mendes de Oliveira, C. 1995, MNRAS, 273, 139

Metcalfe, N., Shanks, T., Fong, R., \& Jones, L. R. 1991, MNRAS, 249,498

Meyer, M. J., Drinkwater, M. J., Phillips, S., \& Couch, W. J. 2001, MNRAS, 324, 343

Moles, M., Marquez, I., \& Sulentic, J. W. 1998, A\&A, 334, 473

Narlikar, J. V. 1977, Ann. Phys., 107, 325

Narlikar, J. V. 1989, Space Sci. Rev., 50, 523

Narlikar, J. V., \& Arp, H. C. 1993, ApJ, 405, 51

Noerdlinger, P. D. 1975, Ap\&SS, 38, 457

Ohyama, Y., Taniguchi, Y., Hibbard, J. E., \& Vacca, W. D. 1999, AJ, 117, 2617

Pecker, J. C., Roberts, A. P., \& Vigier, J. P. 1972, Nature, 237, 227

Pietsch, W., Vogler, A., Kahabka, P., Jain, A., \& Kelin, V. 1994, A\&A, 284,386

Rose, J. A., Gaba, A. E., Caldwell, N., \& Chaboyer, B. 2001, AJ, 121, 793

Roy, S., Kafatos, M., \& Datta, S. 2000, A\&A, 353, 1134
Schlegel, D. J., Finkbeiner, D. P., \& Davis, M. 1998, ApJ, 500, 525

Schombert, J. M., Wallin, J. F., \& Struck-Marcell, C. 1990, AJ, 99, 497

Schneider, P. 1989, A\&A, 221, 221

Sharp, N. A. 1986, ApJ, 302, 245

Sluse, D., Surdej, J., Claeskens, J. F., et al. 2003, A\&A, 397, 539

Smith, J. A., Tucker, D. L., Kent, S., et al. 2002, AJ, 123, 2121

Stockton, A., \& Ridgway, S. E. 1998, AJ, 115, 1340

Sulentic, J. W. 1997, ApJ, 482, 640

Sulentic, J. W., \& Arp, H. C. 1987, ApJ, 319, 687

Sulentic, J. W., Zwitter, T., Marziani, P., \& Dultzin-Hacyan, D. 2000, ApJ, 536, L5

Sulentic, J. W., Rosado, M., Dultzin-Hacyan, D., et al. 2001, AJ, 122, 2993

Sulentic, J. W., Marziani, P., Zamanov, R., et al. 2002, ApJ, 566, L71

Telles, E., \& Terlevich, R. 1995, MNRAS, 275, 1

Teplitz, H. I., Collins, N. R., Gardner, J. P., et al. 2003, ApJS, 146, 209

Tohline, J. E., \& Osterbrock, D. E. 1976, Lick Obs. Bull., 742, 1

Veilleux, S., \& Osterbrock, D. E. 1987, ApJS, 63, 295

Williams, B. A., Yun, M. S., \& Verdes-Montenegro, L. 2002, AJ, 123, 2417

Wolf, E. 1986, Phys. Rev. Lett., 56, 1379

Wu, X. P. 1996, Fund. Cosmic Phys., 17, 1 